\title{
Modeling neurodegenerative diseases with cerebral organoids and other three-dimensional culture systems: focus on Alzheimer's disease
}

\author{
Lalitha Venkataraman $^{1} \cdot$ Summer R. Fair ${ }^{2,3} \cdot$ Craig A. McElroy $^{4} \cdot$ Mark E. Hester $^{1,2,5} \cdot$ Hongjun Fu $^{1}$ (D)
}

Accepted: 25 October 2020 / Published online: 12 November 2020

(C) Springer Science+Business Media, LLC, part of Springer Nature 2020

\begin{abstract}
Many neurodegenerative diseases (NDs) such as Alzheimer's disease, Parkinson's disease, frontotemporal dementia, amyotrophic lateral sclerosis and Huntington's disease, are characterized by the progressive accumulation of abnormal proteinaceous assemblies in specific cell types and regions of the brain, leading to cellular dysfunction and brain damage. Although animal- and in vitro-based studies of NDs have provided the field with an extensive understanding of some of the mechanisms underlying these diseases, findings from these studies have not yielded substantial progress in identifying treatment options for patient populations. This necessitates the development of complementary model systems that are better suited to recapitulate human-specific features of ND pathogenesis. Three-dimensional (3D) culture systems, such as cerebral organoids generated from human induced pluripotent stem cells, hold significant potential to model NDs in a complex, tissue-like environment. In this review, we discuss the advantages of 3D culture systems and 3D modeling of NDs, especially AD and FTD. We also provide an overview of the challenges and limitations of the current 3D culture systems. Finally, we propose a few potential future directions in applying stateof-the-art technologies in 3D culture systems to understand the mechanisms of NDs and to accelerate drug discovery.
\end{abstract}

Keywords Alzheimer's disease $\cdot$ Neurodegenerative diseases $\cdot$ hiPSCs $\cdot 3 \mathrm{D}$ culture $\cdot$ cerebral organoids $\cdot$ tau pathology

\section{Introduction}

This article belongs to the Topical Collection: Special issue on Neurogenesis and Neurodegeneration: Basic Research and Clinic Applications

Guest Editor: Henning Ulrich

Mark E. Hester

Mark.Hester@nationwidechildrens.org

Hongjun $\mathrm{Fu}$

Hongjun.Fu@osumc.edu

1 Department of Neuroscience, The Ohio State University Wexner Medical Center, 616 Biomedical Research Tower, 460 W. 12th Ave, Columbus, OH 43210, USA

2 The Steve and Cindy Rasmussen Institute for Genomic Medicine, Abigail Wexner Research Institute at Nationwide Children's Hospital, 575 Children's Crossroad, Columbus, OH 43215, USA

3 College of Medicine, The Ohio State University Wexner Medical Center, Columbus, OH, USA

4 College of Pharmacy, The Ohio State University, Columbus, $\mathrm{OH}$, USA

5 Department of Pediatrics, The Ohio State University Wexner Medical Center, Columbus, OH, USA
Alzheimer's disease (AD), Parkinson's disease (PD), Frontotemporal dementia (FTD), Amyotrophic lateral sclerosis (ALS) and Huntington disease (HD) are devastating neurodegenerative diseases (NDs) that are prevalent worldwide, particularly in the elderly [1-3]. Although NDs are heterogeneous in their clinical manifestations and mechanisms of cellular vulnerabilities, they share two important common features: 1) an increase in prevalence of disease with age, and 2) the aggregation of cytosolic or nuclear proteins within vulnerable cells and brain regions [3-6]. Protein aggregates such as beta-amyloid $(A \beta)$ plaques, neurofibrillary tangles (NFTs) and aggregates of $\alpha$-synuclein, TAR DNA-binding protein (TDP)-43 and polyglutamine protein are prevalent in these NDs. Furthermore, studies with human brain tissue, animal models and 2D cell cultures have shown that NDs share some common pathological mechanisms that underlie their development and progression, including a defective protein homeostasis network, dysfunctional mitochondrial homeostasis, propagation and seeding of protein aggregates, synaptic toxicity and network dysfunction as well as maladaptive innate immune responses $[3,6,7]$. 
In this review, we discuss the limitations of animal and 2D cell culture models that 3D culture systems such as reproducible hiPSC-derived 3D cerebral organoids (COs) may address. Specifically, we discuss the potential for COs to model crucial features of $A D$, including $A \beta$ and tau pathology, oxidative stress and mitochondrial dysfunction, excitotoxicity, neuroinflammation and neuronal network dysfunction. We then examine the limitations as well as the technical progress being made in $\mathrm{CO}$ generation to overcome these challenges. Finally, we discuss a few potential future directions in applying state-of-the-art technologies in COs to further improve the efficiency of $\mathrm{CO}$ modeling and to understand the underlying disease mechanisms and affected biological pathways and changes in COs in response to various therapeutic drugs (Fig. 1).

\section{COs provide an innovative model of the human brain to understand neurodegenerative disease mechanisms}

Although animal models and 2D neuronal cultures have advanced our mechanistic understanding of neurodegeneration, these models have been unable to recapitulate the complexity of human diseases $[1,8]$. Thus, the lack of biologically relevant model systems hinder progress in discerning disease mechanisms and the development of therapeutic interventions. COs have diverse progenitor and differentiated cell populations that have a spatial organization resembling the fetal neocortex [9-11]. These cell populations are electrophysiologically active displaying periods of coordinated firing, indicating the presence of neuronal networks [12-14]. Compared to studies with analogous monolayer cultures, COs have gene expression profiles that more closely resemble in vivo conditions $[15,16]$. In addition, CO models permit the study of human-specific mechanisms or processes that cannot be easily tested or investigated with animal models. For instance, the developing human brain is comprised of a diversity of progenitor types, including outer radial glia with basal restricted processes that are not represented during mouse corticogenesis [17]. Therefore, model organisms are limited in their ability to recapitulate some disease processes, such as microcephaly [18]. Further, comparative studies of rodent and human mature cortex have demonstrated species divergence in respect to the identities and proportions of neuronal and glial cell types, morphologies, and transcriptomes [19]. These differences suggest that the current understanding of brain pathophysiology, including neurodegenerative disease mechanisms, may be expanded using human-derived models [20].

Despite the advantages of COs, several challenges remain for their use in modeling NDs. Principally, COs exhibit variable morphological and cellular developmental features, which presents challenges in analyzing pathological phenotypes. $\mathrm{CO}$ cell type diversity and relative representation greatly vary by protocol type and culture length [21, 22]. However, recent efforts to enhance the reproducibility of $\mathrm{COs}$ led to various improved protocols. These protocols yield remarkably similar cell type composition in up to $95 \%$ of cultures $[12,23]$. Variability between individual organoids is comparable to the variability in individual human brains which warrants further study [23]. In addition, progenitor zone structures can vary in size and organization among organoids, which may ultimately impact relative cell type presence. To address these challenges, advancements in bioreactor designs and differentiation protocols have increased CO reproducibility [11, 12, 23, 24].

\section{D modeling of AD}

Human COs and other 3D cell culture systems exhibit key neuropathological features of NDs as shown in Fig. 2 and Table 1. Here we focus on 3D modeling of AD-associated neuropathology, oxidative stress and mitochondrial dysfunction, excitotoxicity, neuroinflammation as well as synaptic and network dysfunction.

\section{$A \beta$ pathology}

$\mathrm{AD}$ is characterized by the presence of extracellular $\mathrm{A} \beta$ plaques and intracellular NFTs. Familial AD (FAD) is associated with early onset of $\mathrm{AD}$ symptoms and is caused by variants in the presenilin-1 (PSEN1), presenilin-2 (PSEN2), or amyloid precursor protein (APP) genes, whereas sporadic AD (SAD) is typically associated with multiple genetic polymorphisms and a later onset of symptoms [25]. APP is the precursor of $A \beta$, therefore understanding its processing is important in designing therapeutics that reduce A $\beta$ levels. Sequential APP cleavage with $\alpha$ and $\gamma$-secretase (a complex composed of four components: PSEN1, nicastrin, anterior pharynx defective 1 (APH-1) and presenilin enhancer 2 (PSEN-2)) leads to a soluble $\operatorname{sAPP} \alpha$ fragment under normal conditions [26, 27]. However, APP cleavage via $\beta$ and $\gamma$-secretase results in a $\operatorname{SAPP} \beta$ fragment and $\mathrm{A} \beta$ peptide, the primary component of amyloid plaques in $\mathrm{AD}[26,27]$. Several human clinical trials for $\mathrm{AD}$ have focused on inhibition of $\beta$ and $\gamma$-secretase to decrease $A \beta$ production [28-34]. Although these trials showed a decrease in plasma and CSF A $\beta$ levels, they have failed when it comes to establishing safety and efficacy. These trials have been discontinued based on increased incidence of skin cancer and worsening cognitive scores [29-34].

$3 \mathrm{D}$ cell culture systems overexpressing one or more FAD variants have been used to understand AD pathology [35-37]. Either a thin-layer (100-200 $\mu \mathrm{m})$ or a thick-layer $(\sim 4 \mathrm{~mm}) 3 \mathrm{D}$ culture with Matrigel matrix was developed by introducing viral vectors with FAD variants (Swedish and London 
Mutation/CRISPR Control-

Human iPSC derived

COs/3D Culture

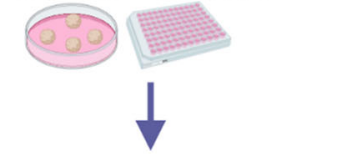

LIMITATIONS

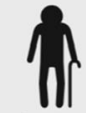

Aging

phenotype

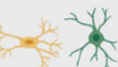

Lack of microglia/

oligodendrocyte

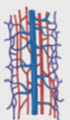

Lack of vasculature

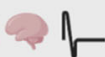

Immature

brain network
CURRENT PROGRESS

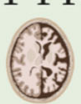

PATHOLOGY

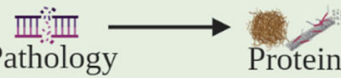

causing mutations aggregation

\section{FUTURE DIRECTION}
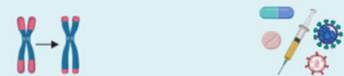

Telomere shortening Drugs/Viruses

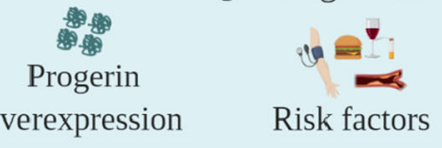

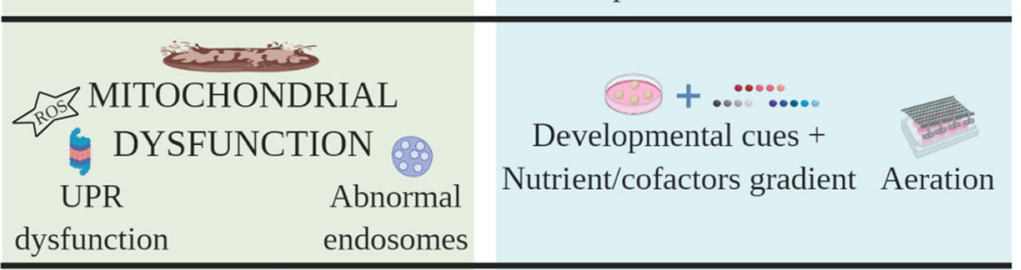

EXCITOTOXICITY

2. $x$

Selective

i.t.

Increase in

vulnerability excitatory neurons

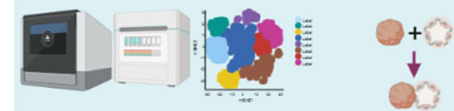

Multi-omics analysis Fusion organoid

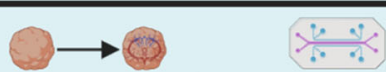

Improved protocol Improved design

NEUROINFLAMMATION
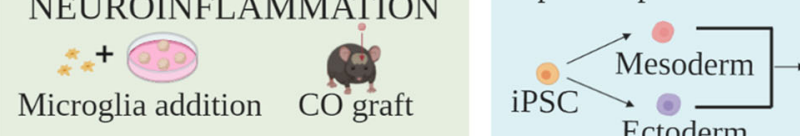

$(4+1)$

Diverse cell populations

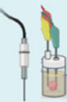

Improved setup/ electrode design

Biocompatible 3D

NETWORK DYSFUNCTION

个excitatory signal

$\checkmark$ inhibitory signal short neurite

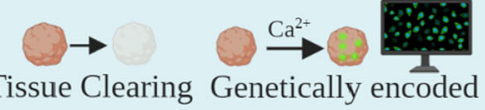

$\mathrm{Ca}^{2+}$ indicators
Fig. 1 Three-dimensional (3D) modeling of neurodegenerative diseases. The overview of limitations, current progress and future direction in using human induced pluripotent stem cells (hiPSCs)-derived 3D cultures/

APPSL and PSEN1 $\triangle 9$ variants) into neural progenitor cells $(\mathrm{ReN})$ [36]. High $\mathrm{A} \beta 42 / 40$ ratios were detected with the presence of SDS-resistant oligomeric $A \beta$ [37] resulting in robust $A \beta$ aggregate deposits (6-8 weeks) [35-37]. 3D cultures with variants ( $\mathrm{I} 45 \mathrm{~F}$ and $\mathrm{I} 47 \mathrm{~F}$ ) in the APP transmembrane domain (TMD), but without the overexpression of the PSEN1 $\triangle 9$ variant, showed either higher (I45F) or lower (I47F) A $\beta 42 / 40$ ratio compared to wild-type (WT) control cultures [37]. Similar to human clinical trials discussed above, application of $\beta$ and $\gamma$-secretase inhibitors in these 3D cultures showed a significant decrease in $A \beta$ pathology [35]. Recent studies indicate that these enzymes have multiple substrates and have cerebral organoids (COs) to model Alzheimer's disease and other neurodegenerative diseases (created with BioRender.com).

several important physiological functions outside the brain including muscle spindle formation [38-40]. Failure of prior clinical trials can potentially be overcome by testing therapies aimed at brain-specific inhibition of $\beta$ and $\gamma$-secretase to minimize off-target effects. $\mathrm{CO}$ model provides an excellent platform to initially validate such therapies.

3D cultures have also been developed with modified hydrogel-based scaffolds [41, 42], such as StarPEG-heparin hydrogel that promotes proliferation of neural stem cells [41]. Upon $A \beta 42$ treatment, the spontaneous electrical activity of these cultures was disrupted, resulting in dystrophic axons, matrix stiffness and decrease in synaptic plasticity [41]. 


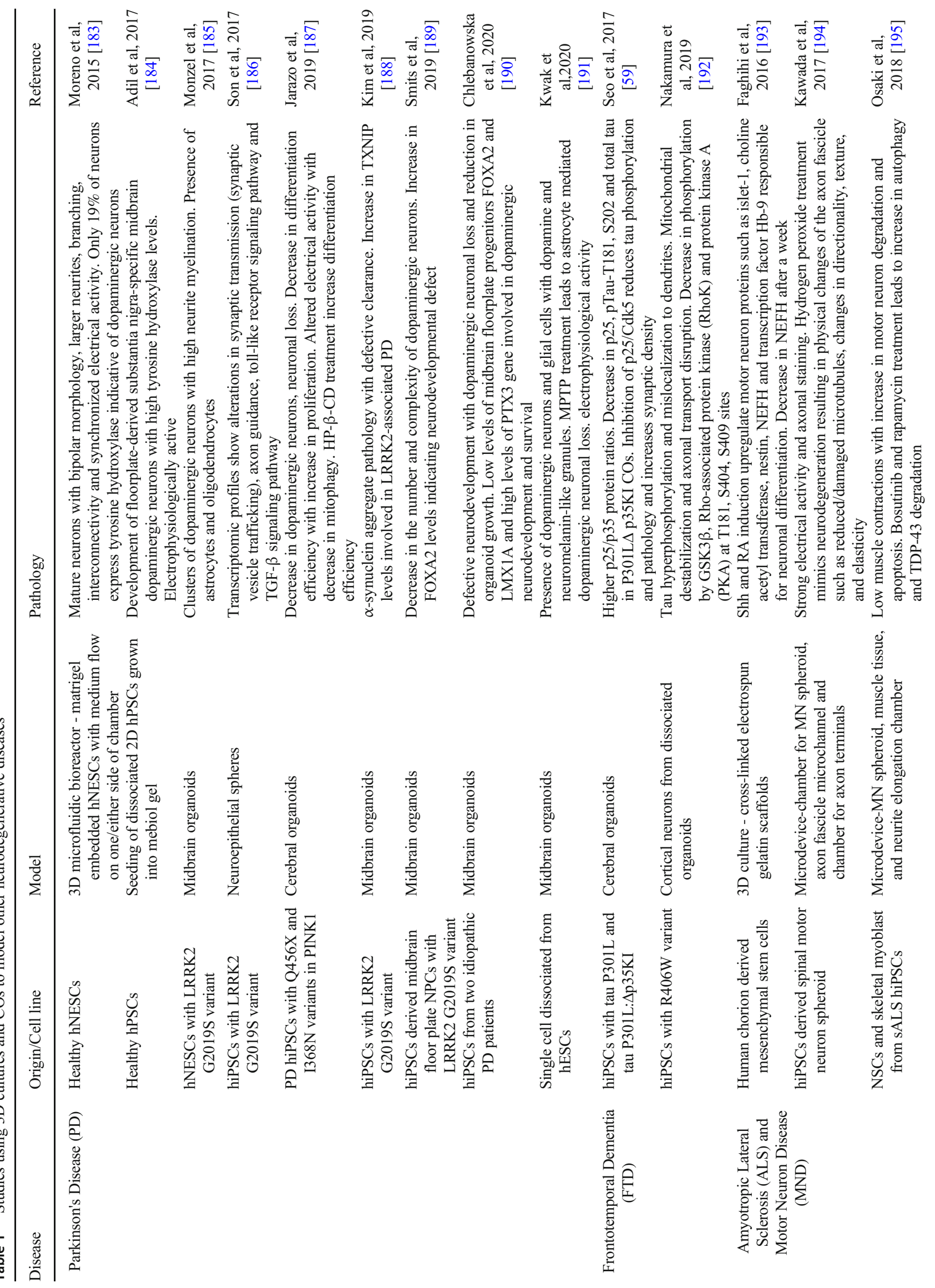




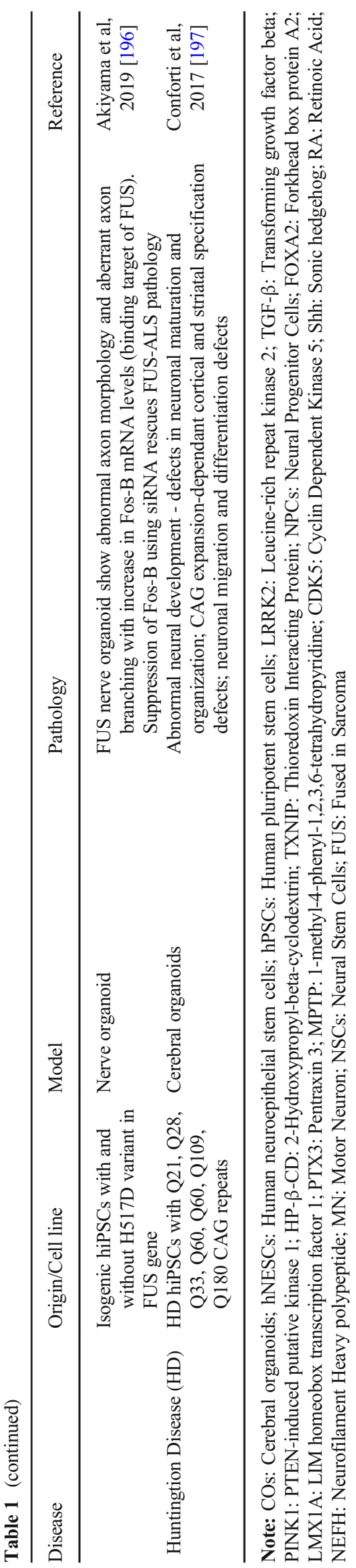

Treatment with IL-4 suppressed this kynurenine aminotransferase -mediated A $\beta$ toxicity [41]. Another model used a silk fibroin porous scaffold with intercalated hydrogel to culture neurons derived from a SAD patient [42]. This culture demonstrated a decrease in spontaneous electrical activity and an increase in $A \beta 42 / 40$ ratio [42].

In contrast, $\mathrm{AD}$-like $\mathrm{CO}$ models are designed to grow in a self-organizing manner [43]. FAD hiPSCs with either APP duplication or variants in PSEN1 (M146I and A264E) and PSEN2 (N141I) have been used to generate such models. These COs exhibited increases in intracellular and extracellular $A \beta$ aggregates consistent with the neuropathology of AD [43, 44] ( [44] published as a preprint in bioRxiv). These characteristics have also been observed in Down Syndrome COs [45, 46]. Patients with Down Syndrome typically present AD pathology very early in life and experience a rapid advancement of the disease progress [47]. This has been attributed to the overexpression of APP on chromosome 21 [47]. Several studies have shown that Down Syndrome and AD-like COs have high levels of diffuse and fibrillar extracellular $A \beta$ deposits as well as increased cell death $[43,45,46]$. As expected, a dramatic decrease in $A \beta$ aggregation was observed when Down Syndrome and AD-like COs were treated with $\beta$ and $\gamma$-secretase inhibitors [43, 45].

Although overexpression of mutant APP, PSEN1 and PSEN2 genes in COs readily models FAD pathology, these variants restrict modeling of SAD pathology. However, healthy COs treated with Aftin-5, an $A \beta-42$ inducer, showed time-dependent increases in both $A \beta 40$ and $A \beta 42$ levels, leading to the formation of $A \beta$ oligomers [48]. This model is able to demonstrate physiological interactions, colocalization and parallel expression of $\mathrm{APP} / \mathrm{A} \beta$ and their high-affinity receptor, cellular prion protein $\left(\operatorname{PrP}^{\mathrm{C}}\right)$, during early phases, indicating a potential role for these proteins in neurodevelopment [48]. This type of chemical induction shows that environmental and other sporadic causes of $\mathrm{AD}$ can also be studied in vitro in CO models.

\section{Tau pathology}

Tauopathies are a spectrum of NDs characterized by abnormal tau hyperphosphorylation and aggregation in the brain. Despite variants identified in the MAPT gene resulting in the formation of paired helical filaments and NFTs in FTD, these variants have not been identified in other tauopathies including AD [37]. Different tauopathies are characterized by the presence of unique, conformational tau strains capable of propagating via anatomically connected brain regions [6]. Here, we review 3D cultures and COs that have been used to model tau hyperphosphorylation, aggregation and its mislocalization, especially in AD and FTD. 

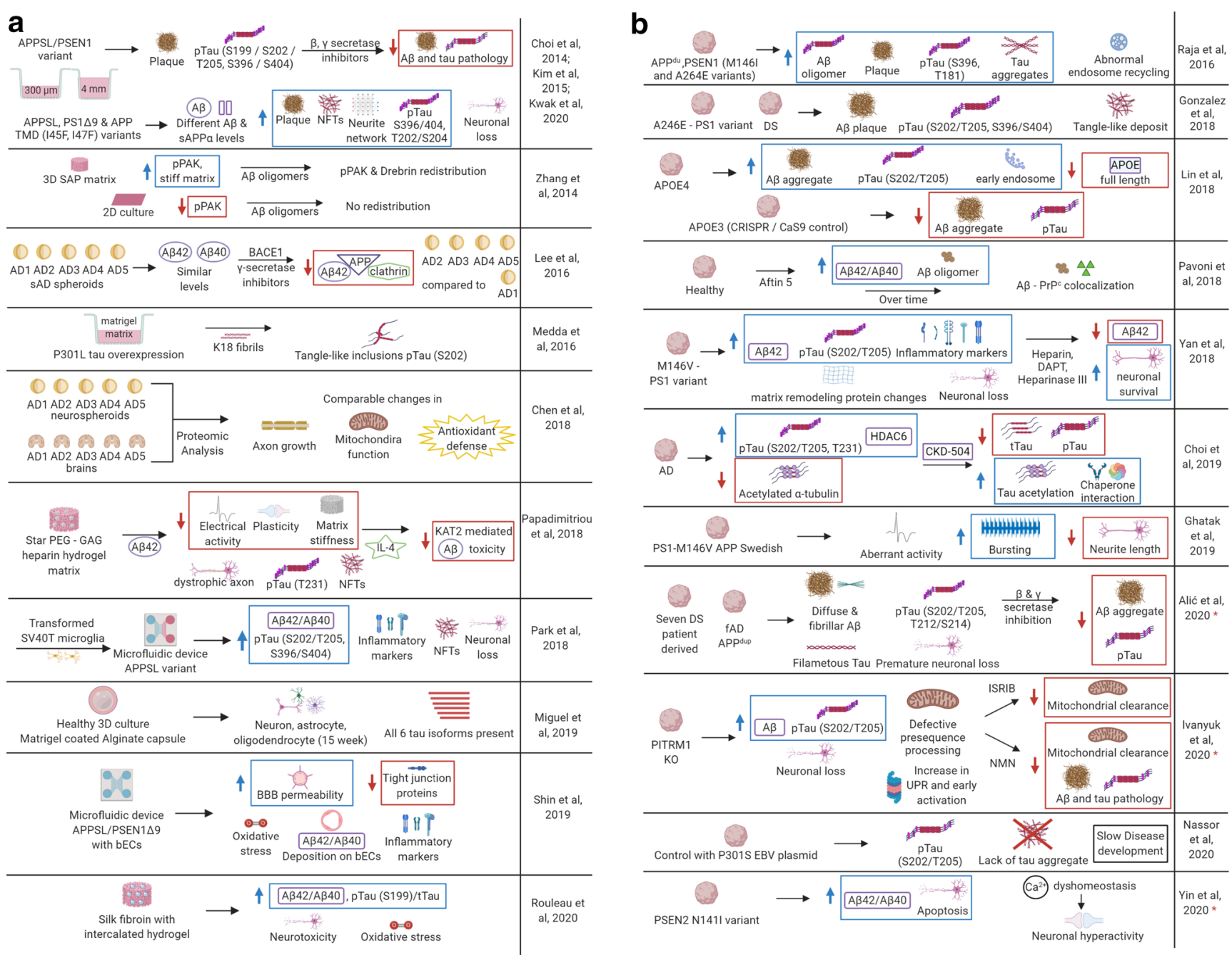

Fig. 2 Three-dimensional (3D) modeling of Alzheimer's disease. Schematic representation of recent studies modeling various aspects of AD using A) 3D cultures, and B) Cerebral organoids. * Studies published as preprints on bioRxiv (created with BioRender.com).

\section{$A D$}

In $\mathrm{AD}$-like $3 \mathrm{D}$ cultures, high $\mathrm{A} \beta 42 / 40$ ratios correlate with high levels of pTau (S396/404, T202/S204, T181, S199/S202/ $\mathrm{T} 205)$, indicating that the $\mathrm{A} \beta 42 / 40$ ratio drives tau pathology [35-37]. Similarly, 3D cultures with variants in the APP transmembrane region showed either high ( $\mathrm{I} 45 \mathrm{~F}$ variant) or low pTau levels (I47F variant) correlating with $A \beta 42 / 40$ ratios [37]. A $\beta 42$ treatment in starPEG-heparin hydrogel-based 3D culture and a silk fibroin-based 3D culture of SAD hiPSCderived neurons also showed an increase in pTau (T231) [41] (S199) [42] levels with Gallyas- and Thioflavin Spositive NFTs [41]. Treatment of these cultures with $\beta$ and $\gamma$-secretase inhibitors caused a decrease in both $A \beta$ and tau pathology [35].

Apart from 3D cultures, COs that overexpress FAD variants exhibit increased levels of pTau-S396, pTau-T181 and Thioflavin S-positive tau aggregates [43]; filamentous tau aggregates (pTau at S202/T205, T212/S214) [45] and cytoplasmic
NFT-like deposits (pTau at S202/T205, S396/S404) [46]. Treatment of the AD-like COs with $\beta$ and $\gamma$-secretase inhibitors reduces $A \beta$ and tau hyperphosphorylation, reiterating that the accumulation of toxic $A \beta$ drives tau pathology [43, 45].

COs have also been used to explore post-translational modifications that may affect the interaction of tau with the molecular chaperones that are responsible for its degradation [49]. For instance, histone deacetylase 6 (HDAC6) is responsible for the deacetylation of $\alpha$-tubulin and tau [50]. HDAC6 levels are elevated in AD and may be involved in the degradation of tau based on HDAC6 involvement in the ubiquitin-proteasome system and autophagy pathway [50]. As expected, decreased levels of acetylated $\alpha$-tubulin and elevated levels of HDAC6 were observed in AD-like COs with pathological tau aggregates compared to control COs [49]. CKD-504, a blood-brain barrier (BBB) penetrating HDAC6 inhibitor, was tested on COs generated from hiPSCs with FAD variants. Total tau and pTau levels were decreased in AD-like COs treated with CKD-504, 
indicating that this inhibitor has the potential to rescue tau pathology [49]. CKD-504 reduced tau levels in the presence of a lysosomal inhibitor but did not degrade tau in the presence of a proteasomal inhibitor, indicating that tau is degraded via a proteasomal pathway. CKD-504 also promoted interactions between tau and chaperones by increasing their acetylation, which further led to proteasomal degradation of tau [49].

In another $\mathrm{CO}$ model, $\mathrm{EBV}$-based plasmids were used to introduce long-term expression of WT tau or mutant P301S tau in hiPSCs. This novel plasmid was shown to be stable for more than 30 passages and did not affect the differentiation of the COs [51]. Unlike overexpression of genes via retroviral approaches, these vectors do not integrate into the genome but remain extrachromosomal and maintain low copy numbers. Although hyperphosphorylated tau (S202/T205) is observed, this model does not exhibit tau aggregation, which is likely due to the requirement of a longer time for disease development [51].

\section{FTD}

FTD is the second leading cause of early-onset dementia and is characterized by a predominant tau, TDP-43 or Fused-inSarcoma pathology $[5,6]$. Here, we focus on 3D cultures and COs derived from FTD patient-derived hiPSCs with MAPT variants that lead to hyperphosphorylation and aggregation of tau.

Alternative splicing of human MAPT in the adult brain results in six tau isoforms $[52,53]$. Due to the lack of an aging phenotype in hiPSC-derived cultures, expressing all six adult tau isoforms is a challenge $[15,54-56]$. This has been partially overcome in a 3D culture model with Matrigel-coated alginate capsules [57]. When BrainPhys medium was used with this model, cortical neurons, astrocytes and oligodendrocytes developed by 15 weeks of culture, whereas they were not all present when standard medium was used even at 25 weeks of culture [57]. All six tau isoforms were expressed when BrainPhys media was used as compared to only five isoforms with standard media. Despite using BrainPhys media, the 2N3R and 2N4R isoforms in this model account for only $1 \%$ and $0.9 \%$ of their prevalence in the adult human brain, respectively [57]. Further studies are required to demonstrate similar ratios of tau isoforms as are expressed in the adult human brain [52, 53]. Another Matrigel-based 3D culture model was characterized by the presence of $3 \mathrm{R}$ tau and the absence of 4R tau [55]. It was demonstrated that 3R tau was less prone to tau aggregation [52] and did not show any pathology when seeded with truncated tau K18 fibrils [55]. However, when mutant tau P301L was overexpressed in the culture, seeding with K18 fibrils resulted in NFT-like inclusions evidenced by the presence of pTau (S202/T205) [55].

Apart from Matrigel-based 3D cultures, COs have also been used to explore the mechanisms underlying FTD [58,
59]. In a recent study, COs derived from FTD hiPSCs with mutant R406W MAPT were dissociated into cortical neurons [58]. To better understand the progression of tau pathology and its phosphorylation, the three major kinases responsible for tau phosphorylation, glycogen synthase kinase 3 $\beta$ (GSK $3 \beta$ ), Rho-associated protein kinase, and protein kinase A were investigated. $\mathrm{R} 406 \mathrm{~W}$ variant cortical neurons showed a decrease in tau phosphorylation by GSK3 $\beta$, Rho-associated Protein Kinase and Protein Kinase A at the T181, S404 and S409 sites of tau, respectively. In particular, inhibition of GSK $3 \beta$ led to an overall increase in tau phosphorylation [58]. Phosphorylation of $\beta$-catenin, another substrate of GSK $3 \beta$, remained unchanged as did phosphatase activity, which can also regulate tau phosphorylation. These results indicate that GSK3 $\beta$ is mainly responsible for the reduced phosphorylation of tau in the R406W variant [58]. Although mutant tau hyperphosphorylation is increased in mouse models and in humans at late stages of the disease, this study indicates that the reduced phosphorylation of tau observed initially is possibly due to the conformational change of tau caused by the variant [58]. Tau mislocalization to the dendrites, mitochondrial destabilization, and disruption of axonal transport are also observed in this model [58]. Although tau hyperphosphorylation occurs ten days after dissociation, further work is required to investigate cellular phenotypes at later time points to further understand the role of the R406W variant in tau pathology and neurodegeneration [58].

To further understand the mechanisms underlying FTD, the role of p25/cyclin-dependent kinase 5 (CDK5) in tau accumulation and the effect of $\mathrm{p} 25$ generation on hyperphosphorylated tau has been examined with tau P301L and P301L: $\Delta$ p35KI isogenic control COs [59]. Cdk5 plays a role in neuronal migration, synapse development as well as synaptic plasticity, and is associated with the regulatory activator p35. High levels of p35 are regulated by either proteasomal degradation or truncation to a soluble $25 \mathrm{kDa}$ form (p25) by calpain [60]. While P301L mutant organoids showed higher $\mathrm{p} 25 / \mathrm{p} 35$ protein ratios, P301L: $\triangle$ p35KI organoids showed significant reductions of p25 and pTau at T181/S202 sites. This finding indicates that $\mathrm{p} 25 / \mathrm{Cdk} 5$ may lead to tau hyperphosphorylation that further increases p25 levels and Cdk5 hyperactivation, forming a feed-forward loop [59]. This model demonstrated that inhibition of p $25 / \mathrm{Cdk} 5$ successfully reduces tau phosphorylation and the pathology associated with FTD [59], which is consistent with previous reports [61]. Further studies are required to understand if kinases like GSK3 $\beta$ are involved in p25/Cdk5mediated tau hyperphosphorylation. GSK3 $\beta$ inhibitors, such as Tideglusib, have been explored in human clinical trials not only for FTD $[62,63]$, but also for AD [64, 65]. Although safety was established, Tideglusib did not produce a significant difference in cognitive scores between treatment and placebo groups. Since these clinical trials were carried out in a small group $(\mathrm{n}=406)$ and for a short duration ( $\mathrm{n}=26$ weeks) [65], 
further dose dependent studies are required in both 3D models and in humans to understand its potential as a therapeutic for AD.

\section{Other pathologies}

Severe Acute Respiratory Syndrome Coronavirus 2 (SARSCoV-2) causes severe upper respiratory tract infection and has also been shown to cause neurological symptoms [66]. Two recent studies have explored the neurological effects of this virus in COs $[67,68]$ ([68] published as preprint in bioRxiv). Day 15 and Day 60 COs were exposed to SARS-CoV-2 [67]. Relatively mature neurons in day 60 COs exhibited substantial SARS-CoV-2 infection in cortical neurons within two days of exposure compared to Day 15 COs [67]. Similar viral load between two- and four-day post infection in COs indicated that the virus did not actively replicate in the COs [67]. Interestingly, the infected COs also showed an increase in early neurodegeneration-like phenotype as pTau (T231) was missorted from the axon to the soma and was colocalized with SARS-CoV-2 [67]. Furthermore, the virus-mediated early tau pathology in COs was shown to be associated with apparent neuronal death [67].

In the second study, SARS-CoV2 infection in healthy nineweek-old COs displayed a significant increase in viral titers from 24 to 96 hours post infection [68]. With an increase in viral titer, an increase in neuronal loss surrounding the infected regions was observed. Single-cell RNA-Seq (scRNA-Seq) of infected COs indicated that genes related to cell division, organelle fission and metabolic processes were enriched following infection [68]. These data also indicated that while SARS-CoV-2-positive cells exhibited upregulation of genes related to viral transcription and hyperoxia, the surrounding SARS-CoV-2-negative cells also displayed upregulated genes related to hypoxia and cell death [68]. This indicates that SARS-CoV-2 has the ability to manipulate host metabolic processes, which may elicit features of neurodegeneration. Thus, these SARS-CoV-2 CO models might be used to study the neuropathology associated with such viruses, and mechanisms underlying accumulation and mis-sorting of tau in different tauopathies including SAD.

Another alternative is to use Traumatic brain injury (TBI) models to study the role of tau in neurodegeneration since it is considered a major risk factor for NDs [69]. A recent study published as a preprint in bioRxiv describes a TBI CO model [70]. In this study, a high intensity ultrasound with a frequency of $510 \mathrm{kHz}$ was applied to healthy COs in $10 \mathrm{~ms}$ bursts for a duration of 2 min to mimic TBI [70]. These COs showed an increase in astrogliosis accompanied by neurodegeneration after the injury [70]. Total RNA-Seq identified changes in genes related to glycolysis, AMPK signaling and protein homeostasis [70]. There was also a significant increase in pTau (T231), pTDP-43 (S409/410) and intracellular calcium levels
[70]. Previous studies in FTD and other NDs have identified dysregulation of nuclear pore proteins which can form pathological aggregates with tau and TDP-43 [71]. Similar disruption of the nuclear pore protein, Nup98, was observed in this TBI CO model [70]. Thus, this model can be used to study ND mechanisms involving nuclear pore protein dysregulation, TDP-43, and tau phosphorylation.

\section{Oxidative stress and mitochondrial dysfunction}

Generation of reactive oxygen species (ROS) and mitochondrial dysfunction are early features of AD that lead to an increase in cell toxicity and neuronal loss [72]. These signatures have been readily demonstrated in comparative proteomic analyses of AD neuro-spheroids and post mortem AD brain tissue [73]. This is an important reflection of the potential of similar in vitro systems to recapitulate and investigate key protein signatures that underlie AD pathogenesis [73].

Pitrilysin metallopeptidase 1 (PITRM1) is responsible for the processing and degradation of mitochondrial precursor proteins [74]. PITRM1 dysfunction causes improper degradation and over-accumulation of $\mathrm{A} \beta$ in the mitochondria, resulting in mitochondrial dysfunction and neurodegeneration [74]. Since PITRM1 deficiency can cause embryonic lethality in mice, mechanisms underlying $A \beta$ accumulation were investigated using human PITRM1 knockout COs (published as a preprint in bioRxiv) [75]. Increases in APP, A $\beta 40, A \beta 42$, the A $\beta 42 / A \beta 40$ ratio, plaque-like amyloid aggregates and pTauS202/T205 levels were detected in PITRM1 KO COs compared to controls. In addition, increases in ubiquitinated proteins, mitochondrial unfolded protein response (UPR) transcripts and upregulation of mitochondrial proteases were detected in PITRM1 KO COs compared to control COs. These findings indicate that PITRM1 is essential for maintaining mitochondrial protein homeostasis by degrading target sequences like $A \beta$ and possibly tau [75].

Mitochondrial UPR inhibition via integrated stress response inhibitor (ISRIB) resulted in increases of APP, $\mathrm{A} \beta 42 / \mathrm{A} \beta 40$ and $\mathrm{pTau}$ in PITRM1 KO COs, suggesting a decrease in mitochondrial clearance [75]. Treatment with the NAD+ precursor, nicotinamide mononucleotide, which can improve mitochondrial function and clearance [76], rescued the $A \beta$ and tau pathology in PITRM1 KO COs [75]. This indicates that mitochondrial UPR activation and mitochondrial clearance may play an important protective role in attenuating AD-like phenotypes induced by PITRM1 deficiency. This CO model provides a link between mitochondrial dysfunction and neurodegeneration in $\mathrm{AD}$.

Abnormal endosome recycling and morphology is another related characteristic feature of AD [77, 78]. Endosomes are involved in the formation and engulfment of autophagic 
substrates around damaged mitochondria during Parkinmediated mitophagy $[79,80]$. COs with FAD variants show abnormal endosome morphology and a significant increase in large endosomes $(1-2 \mu \mathrm{m})$ compared to control organoids [43]. Uptake of labelled transferrin via clathrin-mediated endocytosis can be used to assess the functionality of endosomes. There is a significant increase in the size of transferrin-containing endosomes in AD-like COs indicating abnormal endosome recycling [43]. Thus, COs can be employed to investigate the mechanisms underlying mitochondrial dysfunction and cell death in NDs, especially AD.

\section{Excitotoxicity}

Certain neuronal subpopulations are selectively susceptible to protein aggregation and neuronal loss in $\mathrm{AD}$ and other NDs [4]. COs with a PSEN1 M146V variant exhibit an imbalance in excitatory and inhibitory neurons [81]. COs derived from mouse iPSCs also show an increase in glutamatergic neurons [82]. Analyses of COs derived from AD hiPSCs (with different variants) are needed to detect changes in cell-type composition and neurotransmitter properties. Further analysis of AD-like and control COs with scRNA-Seq and complementary immunohistochemistry will be especially critical to determine the gene signatures responsible for selective cellular vulnerability and excitotoxicity in AD.

\section{Neuroinflammation}

Accumulation of aggregated $A \beta$ and hyperphosphorylated tau can trigger an immune response, mediated by the release of pro-inflammatory cytokines, contributing to the progression of $\mathrm{AD}$ [72]. Despite the lack of microglia, neuroinflammation has been observed in COs with a PSEN1 M146V variant [81]. Elevated gene expression of proinflammatory cytokines such as IL- 6 and TNF- $\alpha$ has been observed in AD-like COs compared to controls [81]. Increased LDH activity was also detected, indicating increased cytotoxicity and neuronal loss [81]. Treatment of AD-like COs with molecules such as DAPT (a $\gamma$ secretase inhibitor), heparin (to compete with heparan sulfate proteoglycan -A $\beta 42$ binding), and heparinase III (to digest heparin sulfate proteoglycan) showed antiinflammatory effects with reduced toxicity and decreased A $\beta$ levels [81]. While DAPT increased cell survival in $\mathrm{AD}$-like COs, no change was observed with heparin or heparinase III treatment, which may be due to their large size and inefficient diffusion [81].

As detailed below, COs lack microglia, so one strategy to overcome this lack of microglia in 3D models has been addressed using an engineered microfluidic platform [83]. This approach involves the use of chambers for microglia to model the interactions of $\mathrm{AD}$ neurons, astrocytes, and microglia. The neurons and astrocytes in the microfluidic platform showed higher differentiation potential and stronger functional electrical activity compared to 2D cultures [83]. This model allows the observation of microglia recruitment, release of cytokines (IL8, TNF- $\alpha$ and IFN- $\gamma$ ), chemokines (CCL2, CXCL10 and CX3CL1) and the neuroinflammatory processes involved in neurodegeneration [83]. Primary human microglia transformed with simian virus 40 have been used in this model [83], and further studies are required with hiPSC-derived microglia and human primary microglia to replicate these findings.

\section{Neural circuit dysfunction}

Synaptic dysfunction and associated network abnormalities occurring before overt neurodegeneration are implicated as an early pathological event in $\mathrm{AD}$ and are believed to contribute to the hyperexcitability in $\mathrm{AD}$ as well as other NDs [84-88]. To understand the mechanisms underlying neuroelectrical deficits in $\mathrm{AD}$, $\mathrm{COs}$ were derived from AD hiPSCs with either a PSEN1 variant (M146V) or the APP Swedish variant [89]. Electrical activity was measured using microelectrode array (MEA) in both 2D cultures and COs, and AD-like COs showed enhanced excitability with shortened neurites compared to control COs [89]. In 2D neuronal culture, increases in the frequency and amplitude of spontaneous excitatory post synaptic currents and a decrease in inhibitory signals in AD-like neurons have also been shown to contribute to network imbalance [89]. This model recapitulates aspects of early $\mathrm{AD}$ pathophysiology with changes observed in neuronal morphology and synaptic transmission contributing to aberrant electrical activity, i.e. hyperexcitability [89]. Further studies are required to assess changes in excitatory and inhibitory neuronal subpopulations, synaptic activity, and circuitry function in AD-like COs.

\section{D microenvironment abnormality}

The BBB is composed of pericytes, astrocytes and endothelial cells (bECs), which form functional tight junctions to separate brain tissue from peripheral blood and provide a defense against disease-causing pathogens and toxins that may be present in peripheral blood [90]. This highly selective semipermeable membrane becomes dysfunctional due to endothelial and pericyte degeneration in $\mathrm{AD}$ [90]. An AD microfluidic platform derived from a ReN cell line with FAD variants has been used to study BBB dysfunction [91]. Increased BBB permeability and decreased tight junctions and adheren proteins were observed in this AD-like model which could be reversed with the drug Etodolac [91]. 
The importance of the mechanical environment in mimicking AD pathology has been further explored by investigating P21 activated kinases (PAKs) in 3D culture [92]. PAKs convert mechanical stimuli such as the mechanical properties of a 3D matrix to chemical stimuli in a cell [93]. PAKs translocate from the cytosol to the cell membrane in hippocampal neurons treated with $\mathrm{A} \beta$ oligomers [93]. Cofilin, an actin-regulatory protein, has a reciprocal relationship with both $\mathrm{pPAK}$ and Drebrin, an actin-stabilizing protein [93]. PAKs have thus been implicated in $\mathrm{AD}$, especially in cytoskeleton dynamics and neuronal morphology [93] which has been investigated with a 3D self-assembling peptide (SAP) matrix [92]. As expected, pPAK and Drebrin levels were significantly higher in $3 \mathrm{D}$ compared to $2 \mathrm{D}$ cultures, with relatively lower levels of Cofilin [92]. Higher co-localization of F-Actin and Drebrin was observed in 3D cultures, however, it could not be recapitulated in 2D cultures [92]. These differences could be due to the SAP matrix that provides a $3 \mathrm{D}$ scaffold recapitulating the native brain tissue environment. The effects of $A \beta$ oligomer treatment on the PAK-cofilin signaling pathway has also been studied in 2D and 3D cultures [92]. While no difference has been observed between control and $\mathrm{A} \beta$ treatment in $2 \mathrm{D}$ cultures, there was a depletion of activated PAK, a redistribution of pPAK to the submembranous region, and the presence of fragmental pPAK in 3D cultures [92]. This study shows that compared to $2 \mathrm{D}$ cultures, the $3 \mathrm{D}$ culture environment offers a more suitable mechanical environment that recapitulates the $\mathrm{AD}$ phenotype and serves as a more relevant in-vitro model for studying the disease.

Changes in the extracellular matrix (ECM) have also been investigated using COs generated from hiPSCs with the PSEN1 M146V variant [81]. Altered levels of ECM molecules like Syndecan-3 and matrix metalloproteinases have been observed in AD-like COs, indicating that changes in ECM composition and 3D environment remodeling is possibly associated with the AD phenotype [81].

\section{Challenges/Limitations}

Despite the many advantages associated with modeling NDs using COs, several challenges still exist. This section details the major drawbacks associated with COs, including the lack of aging, cell heterogeneity, vasculature, mature synaptic connection, and brain networks. We also discuss how these challenges are being addressed and point out areas that warrant further study.

\section{Aging}

One current challenge is that hiPSC-derived neuronal cultures do not express all six tau isoforms expressed in the adult brain. These neurons express high levels of embryonic 3R tau and low levels of the $4 \mathrm{R}$ tau isoforms [15, 54, 55, 94]. Although this issue has been overcome in a $3 \mathrm{D}$ culture with both $3 \mathrm{R}$ and $4 \mathrm{R}$ tau expression [57], this model does not mimic the ratios of human adult tau isoform expressed in vivo. COs also show fetal neocortex properties making it difficult to model NDs $[10,15,95,96]$. Additionally, there is a loss of agingassociated markers such as DNA damage and mitochondrial stress in COs [97]. This can potentially be overcome by using the SARS-CoV2 virus, or ZIKV virus, which elicit neurodegenerative phenotypes in COs that mimic age-related deficits as seen in $\mathrm{AD}[5,67,98,99]$. These viruses may therefore be used to model pathological features associated with neurodegeneration. Alternatively, drugs like Aftin-5, rotenone and hypoxic culture conditions can be used to induce $A \beta$ and other aging disease related pathologies in COs $[48,100,101]$.

Telomerase manipulation or overexpression of progerin [97, 102] can also be used to model aging. Progeria is caused by a variant in the Lamin $\mathrm{A} / \mathrm{C}$ gene, which causes accelerated premature aging [103]. Progerin overexpression in hiPSCderived PD neurons results in the accumulation of ROS, shorter dendrites, DNA damage and loss of tyrosine hydroxylase expression [97]. Telomere shortening is another tool that has proven effective in inducing aging and disease-specific phenotypes such as increase in mitochondrial stress and reduction in the number of dendrites [102]. While artificial aging might not replicate all the real aging phenotypes in human NDs, it provides a more relevant platform for modeling AD and other NDs.

\section{Microglia and oligodendrocytes}

Occurrence of different cell types in COs such as neural progenitor cells, radial glia, astrocytes, and oligodendrocytes largely depend on the culturing protocol. Longer culture times increase the complexity and diversity of cell types in COs [95]. While cell types within COs are derived from the neuroectodermal lineage, other brain-resident cell types such as microglia are derived from the mesodermal lineage, which accounts for the absence of microglia in COs. Although modifying the differentiation protocol results in innate microglia, there is also an increase in organoid variability with differences in microglial distribution between organoids [104]. The lack of microglia and reduced astrocytes in COs can be overcome by transplantation of human astrocytes and microglia derived from hiPSCs [105-108] into COs, which enhances the frequency of synchronized burst activity and $\mathrm{CO}$ development. This type of co-culture is stable long-term and provides a platform to model neuroinflammation and neuron-glia interactions. Methods have also been recently developed to increase the proportion and maturation of oligodendrocytes in COs. In a recent study, COs were exposed to a combination of small molecules and growth factors at defined time points to modulate SHH and WNT signaling and also promote 
oligodendrocyte development and maturation [109]. Additional methods to increase the percentage of oligodendrocytes in COs involve using growth factors such as IGF-1 and PDGF-AA and pro-myelination drugs [110].

Although scRNA-Seq of COs shows the presence of broad classes of cell types, the culture environment may induce ER stress, which in turn affects cellular subtype differentiation [21]. Generation of a heterogenous cellular population in COs involves the use of neural induction and patterning cues along a 3D printed biocompatible scaffold, capable of providing a suitable microenvironment for $\mathrm{CO}$ development $[81,92$, 111]. One of the major challenges in the field is optimization of differentiation protocols to increase cellular heterogeneity, while reducing the stress and variability associated with organoid culture. One strategy to overcome this challenge is by using a 3D accoustofluidic device to model neuroinflammation in AD [112]. Piezoelectric transducers in the device can manipulate the neurospheroids to model $A \beta$ toxicity, microglia recruitment and activation in $\mathrm{AD}$ [112]. Lack of microglia and astrocytes and the inability to model brain regions specifically affected by $\mathrm{AD}$ can be overcome by fusing two organoids to form a fusion organoid or assembloid $[96,113]$. This can be used to model the inter-neuron migration and neuroinflammation underlying AD.

\section{Vasculature}

COs do not have inherent functional vasculature, which may lead to the formation of necrotic cores as their central most region does not receive sufficient nutrients and oxygen supply $[43,111,114,115]$. Despite the use of miniaturized, multiwell spinning bioreactors which increase diffusion of nutrients and oxygen within COs, oversized COs still lack uniformity and display a necrotic core [116]. This limits the size of organoids that can be grown in vitro without functional vasculature.

COs co-cultured with endothelial cells show vascularization on the exterior but no vascularization in the core of the organoid [117]. Human E26 transforming sequence variant 2 (ETV2) is a transcription factor responsible for the development of vasculature in embryos [118]. Ectopic expression of human ETV2 in COs results in the formation of fetal neocortex-like vasculature [119]. Another alternative is an organoid graft transplanted into a mouse brain, which becomes vascularized with the development of blood vessels, integration of microglia, neuronal maturation and axonal growth extending from the organoid graft into the host [120]. However, in another study, a similar organoid graft into a mouse brain $(\mathrm{n}=1)$ did not survive [117], indicating the need for additional studies to assess the viability and development of vasculature in such grafts.

One important aspect of brain vasculature is the formation of the BBB [90]. Several microfluidic devices successfully model the BBB and overcome the lack of vasculature in COs. Despite changes in the microenvironment and BBB permeability, this BBB model lacks astrocytes and pericytes which play an important role in the function of the BBB [91]. To overcome this issue, some models have incorporated rat astrocytes [121, 122] and hiPSC-derived pericytes [123] with endothelial cells, astrocytes, and neurons to model the $\mathrm{BBB}$. These BBB models show in vivo BBB characteristics with high transendothelial electrical resistance and expression of tight junction proteins [121-126]. Despite these advances, the BBB platform and differentiation protocols still require optimization to develop functional vasculature in COs.

\section{Neural circuits}

COs fail to establish mature neural networks that can model NDs. COs transition from initial irregular activity to synchronous oscillatory patterns. As the COs develop inhibitory neurons, there is complex spatiotemporal variation in network activity [127]. However, the electrical activity observed in COs is very similar to preterm human EEGs [127], indicating the lack of maturity.

\section{Applications and Future Direction}

COs offer several advantages over animal models and 2D cultures, especially in terms of studying human-specific disease initiating events, ease of genetic and environmental manipulation, and applicability to high throughput assays for drug discovery. In this section, we discuss applications of COs and explore avenues that need further research to better understand NDs.

\section{Multi-omics Analysis}

Multi-omics studies leverage collective insights from transcriptomics, proteomics, and metabolomics. Total and single-cell multi-omics analyses have been useful in resolving distinct cellular architectures and functions of heterogeneous populations in COs. They can further aid in the elucidation of the relationship between observed phenotypes in NDs and their underlying disease mechanisms. Such analyses may also accelerate advances in the fields of personalized diagnostics and therapeutics.

\section{Transcriptomics}

The transcriptomic identities of COs and other 3D culture model systems have been extensively studied $[12,21-23$, 95, 127]. Cumulatively, these reports demonstrate that the transcriptome can be readily interrogated to understand the 
etiology of NDs and evaluate treatment responses at the RNA level.

Several total RNA sequencing (RNA-Seq) analyses have been performed to validate 3D ND models and discern their disease characteristics. For instance, the transcriptome of a 3D starPEG-Heparin-hydrogel-based culture of human fetal primary astrocytes exhibit several in-vivo signatures such as enrichment of transcripts related to mature neuronal physiology as well as synapse and axon development when compared to an equivalent $2 \mathrm{D}$ model [41]. In the same study, $\mathrm{A} \beta 42$ treatment in primary and induced $3 \mathrm{D}$ cultures resulted in impaired plasticity and network formation, though via distinct molecular mechanisms [41]. These findings underscore the important considerations in experimental design, such as the limitations in molecular programing that induced 3D models may have compared to analogous primary or self-organizing $\mathrm{CO}$ models.

Total RNA-Seq analyses have also been carried out in APOE3 and APOE4 COs. A recent transcriptomic study implicated the loss of transcriptional repression by Repressor Element 1-Silencing Transcription factor (REST) in the pathogenicity of APOE4 with 3D SAD models [128]. In comparison to APOE3 COs, APOE4 COs exhibit accelerated neuronal differentiation at the mRNA level, as evidenced by their enrichment of genes -associated with nervous system development, synaptic transmission, and neurogenesis [128]. Interestingly, APOE4 COs also exhibit increased total tau expression and pTau-Ser202 [128]. These altered transcriptomic phenotypes appear to be, in part, a reflection of increased activity of REST targets. This mechanism extends to 3D SAD models and provides a more physiologically relevant platform to appreciate the potential importance of transcriptional regulators such as REST in the etiology of NDs.

While total RNA-Seq studies allow for the identification of large-scale changes in transcriptomic profiles, the advent of scRNA-Seq has made it possible to appreciate transcriptionally distinct and diverse cell populations [22, 23, 129]. Given that the human brain and COs are intrinsically heterogenous structures, the resolution of specific cell types at the transcriptomic level is important. An expansive scRNA-Seq study of over 80 thousand single cells from nearly $50 \mathrm{AD}$ patient prefrontal cortex samples revealed that $\mathrm{AD}$ has high cell-type specificity in early pathogenesis that later manifest as global stress response [130]. There are also sex-specific trends in $\mathrm{AD}$ indicated by transcriptomic alterations in particular cell types [130], which may lend an important insight to the selection of iPSC donors for AD CO models. Novel diseasespecific deficits in neuronal differentiation, neurodevelopment and neurotransmission have been identified via scRNA-Seq in $\mathrm{CO}$ models for bipolar disorder, schizophrenia and autism spectrum disorder (ASD) [131-133]. Although these studies may not be directly generalizable to neurodegenerative conditions, they exemplify an ideal strategy to detect disease-specific transcriptomic changes at the cellular level, which may be of great benefit to further our understanding of ND mechanisms.

The ability to detect disease- and cell-type-specific molecular signatures of NDs with scRNA-Seq can be of additional value in spatial transcriptomic applications. Spatial transcriptomics methods provide an important morphological context to reconstruct the physiologic organization of expression patterns [134]. It has been used to refine inter-glia activity implicated in the $A \beta$ plaque accumulations of $A D$ pathogenesis present in rodent models [135]. This application of spatial transcriptomics is particularly interesting as it permits the specific resolution of pathogenic features in the vicinity of plaque accumulations. If adapted for use in COs, this method holds great potential to study the humanspecific features of $\mathrm{AD}$ pathogenesis as they progress with relative spatial organization.

\section{Proteomics}

Proteomics provides a functional map of all the proteins within a cell or tissue. Proteomic analysis of 3D neuro-spheroids derived from five AD patients exhibited differences in the expression of proteins related to axon growth, mitochondrial function, and antioxidant defense compared to controls [73]. Analysis of AD post-mortem brain tissue also revealed differences in proteins related to axonal injury and transport, oxidative stress, and inflammation [73]. The overlap in the differentially expressed proteins between AD postmortem brain tissue and the 3D AD neuro-spheroids indicates that $3 \mathrm{D}$ models can recapitulate at least some of the AD-like phenotypes [73]. Another study involved proteomic analysis of the CA1 and subiculum regions of human postmortem AD brains $(n=40)$ [136]. Analysis revealed an increase in proteins related to astrocyte, ECM, cytoskeleton and synaptic environment over the Braak stages [136]. A similar study of the olfactory bulb in human postmortem AD brains ( $n=4-5 /$ group) revealed impaired neuritogenesis, synaptic plasticity and protein degradation [137]. Further analysis of the different Braak stages also revealed cytoskeletal dysregulation followed by mitochondrial impairment and unstable RNA transcripts [137]. Such proteomic analyses of postmortem human brains and 3D ND models shed light on the early neurodegenerative mechanisms aiding in the identification of potential biomarker and therapeutic candidates.

Proteomics has also been used to assess drug efficacy by measuring changes in the proteome after drug treatment. For example, one study demonstrated that the treatment of BACE1 and $\gamma$-secretase inhibitors significantly reduced A $\beta 42$ levels in four out of five 3D AD neuro-spheroids [138]. Proteomic analysis of these neuro-spheroids with lower A $\beta 42$ levels revealed a $30-40 \%$ reduction of APP protein 
(BACE1 substrate) and clathrin (APP endocytosis) compared to the $\mathrm{AD}$ neuro-spheroid with high $\mathrm{A} \beta 42$ levels [138].

In another study, the proteomic changes in Down Syndrome COs was measured before and after treatment with harmine, a $\beta$-carboline alkaloid and an inhibitor of chromosome 21 encoded protein kinase (dual specificity tyrosine-phosphorylation-regulated kinase 1A, DYRK1A) [139]. The Down Syndrome organoids exhibited dysregulation of neurogenesis, axon guidance and extracellular matrix remodeling. After the treatment with harmine, there was a partial restoration of key regulators of cortical development, transcription factors and normalization of ECM proteins [139].

Another proteomics study analyzed COs treated with 5methoxy-N, N-dimethyltryptamine, a traditional psychedelic drug associated with cognitive gain and structural changes related to attention in the brain $[140,141]$. Differential expression of proteins associated with long-term potentiation, dendritic spine formation, microtubule dynamics and cytoskeletal reorganization was observed [141]. There was also a decrease in cell death, suggesting that the drug confers a protective effect [141]. These results indicate the potential of proteomics in identifying subtle differences in protein expression after drug treatment, which could help in elucidating the mechanistic changes induced by drug treatment and could pave the way for personalized medicine in the future.

\section{Metabolomics}

Metabolomics is the study of the metabolome, which generally refers to all small molecules from a biological sample with a molecular weight of less than $1.5 \mathrm{kDa}$. It provides a snapshot of the interaction between genetic and environmental factors. Since metabolites are the end products of the cellular regulatory processes, metabolomics is regarded as the omics field most closely related to phenotype [142-144]. Therefore, it holds great potential for the discovery of ND-relevant biomarkers and therapeutic targets. Although to date, there have been no reports of metabolomics profiles in COs generated from patients with NDs, metabolomics has been applied to AD patient samples with some success. In one such study, the authors utilized untargeted metabolomics of cerebrospinal fluid (CSF) from control subjects or subjects with stable mild cognitive impairment (MCI), MCI that progressed into $\mathrm{AD}$, or $\mathrm{AD}$ and identified 17 biomarkers capable of predicting the development of AD with an accuracy of $98.7 \%$ [145]. This and other metabolomics studies [146-150] of AD patient samples not only provide great potential for developing methods for early diagnosis in $\mathrm{AD}$, but also provide a potential mechanism to validate AD-like COs and other ND organoid models to ensure that they recapitulate the phenotypes seen in humans.
Metabolomics studies on organoid models of other diseases demonstrate the promise of the method for discovery in NDs. The CLN3 ${ }^{\mathrm{Q} 352 \mathrm{X}}$ variant was identified in a juvenile form of neuronal ceroid lipofuscinosis (JNCL), a lysosomal storage disorder that causes pediatric neurodegeneration. Decreases in metabolites like creatinine and gammaamino butyric acid (GABA) were observed in CLN3 ${ }^{\mathrm{Q} 352 \mathrm{X}}$ organoids compared to isogenic control organoids [151]. Transcriptomics and metabolomics data showed that the CLN3 ${ }^{\mathrm{Q} 352 \mathrm{X}}$ variant caused neurodevelopmental changes and neurodegeneration in JNCL [151].

Metabolic profiles have also been generated from healthy 3D neurospheres at 2, 4 and 8 weeks of differentiation [100]. Changes in the metabolic profile are consistent with the different stages of differentiation [100]. After the treatment of $5 \mu \mathrm{M}$ rotenone, which interferes with the electron transport chain in mitochondria, analysis of the metabolites confirmed mitochondrial dysfunction, disruption of gene regulation by peroxisome proliferation and defective nervous system development [100]. Thus, metabolomics offers great potential for assessing drug efficacy, elucidating underlying disease mechanisms and discovery of clinically relevant biomarkers in COs modeling NDs.

\section{Propagation and spread of pathogenic protein aggregates}

Protein misfolding, aggregation, and cell-to-cell propagation of these protein aggregates is believed to play important roles in the pathogenesis of many NDs. These processes have been modeled in animals, cell culture, and human postmortem brain tissue $[6,7,152]$. To our knowledge, COs have not been used to model the propagation and spread of $\mathrm{A} \beta$ and $\mathrm{pTau}$. However, COs have been used to model propagation in Creutzfeldt-Jakob Disease (CJD), a type of prion disease in which there is abnormal buildup of prion protein, which propagates from cell to cell in an infectious manner causing dementia [153]. In this study, COs were inoculated with two different conformational CJD subtypes MV1 or MV2 (Methionine-Valine type 1 and 2) [153]. MV2 subtype showed higher seeding activity than the MV1 subtype. Even though seeding caused by the initial inoculum was cleared, a resurgence of prion seeding activity was detected in the COs indicating de-novo propagation and spread [153]. Modeling prion propagation in COs provides encouraging evidence that the propagation and spread of other conformational proteins such as pTau and $A \beta$ isolated from patients with AD and FTD may be possible in COs as well.

Although literature on propagation and spread in COs is still lacking, 2D cultured neurons derived from hiPSCs have been used to investigate the spread of intermediate protein strains in AD. Tau repeat domain (tau-RD) with P301L and V337M (tau-RD-LM) variants introduced into hiPSC-derived 
neurons have shown AD-like aggregation of tau. Tau seeds extracted from this culture have shown the ability to recapitulate and induce tau aggregation in a non-transduced neuronal culture [154]. This indicates that the extracted pathological tau seeds can cause tau aggregation via cell-to-cell propagation and similar studies should be replicated in COs.

$A \beta$ and tau oligomers are intermediate protein species that are toxic in AD [155]. Mechanisms underlying the pathogenicity and spread of these species have been investigated in $2 \mathrm{D}$ cultures. In one study, neurons derived from healthy hiPSCs were treated with either full-length tau monomers or oligomers. While there was no significant difference between the monomer treatment and control, neurons treated with tau oligomers recruited endogenous tau and showed an increase in pTau (T231, S396/404) aggregation, resulting in progressive neurite degeneration and neuronal loss [156]. Tau oligomer treatment has also shown a reduction in synaptic integrity, neurotransmitter release, and calcium signaling [156], which is in agreement with the toxicity induced by tau oligomers in primary neurons and AD-like animals [155, 157-159].

Another study analyzed the propagation of AD brainderived oligomeric $A \beta(O A \beta)$ via exosomes in neurons differentiated from the SHSY5Y neuroblastoma cell line and the neural stem cell line (AF22) derived from healthy hiPSCs [160]. Neurons incubated with oA $\beta$-rich exosomes (donor cells) and co-cultured with recipient neurons showed the presence of $\mathrm{oA} \beta$ in both neuronal types [160]. When secondary neurons were further co-cultured with untreated SHSY5Y neurons, they also showed the presence of oA $\beta$, indicating that unexposed neurons take up and propagate intact exosomes rich in $\mathrm{oA} \beta[160]$. Inhibition of exosome secretion by siRNA significantly reduced oA $\beta$ transfer between neurons. A $97.7 \%$ reduction in exosome uptake by the recipient cells was observed when neurons were treated with Dynasore (a dynamin inhibitor) [160]. This indicates that dynamin is likely involved in the propagation and spread of oA $\beta$ [160]. These results need to be replicated in COs since they provide a more complex environment to study the propagation of protein aggregates [8]. Other mechanisms underlying the propagation of aggregated proteins such as receptor mediated, clathrin dependent and independent endocytosis [161] also need to be explored in COs.

\section{Neuronal and regional cellular vulnerability}

Neuronal sub-populations show differential vulnerability to different pathological protein aggregates found in NDs, which cumulatively aggregate and thereby affect distinct regions of the brain $[3,4,6]$. Mechanisms underlying this 'selective vulnerability' are important to understanding disease etiology and designing targeted therapeutic strategies. The APOE gene encodes for the apolipoprotein E protein and the E4 variant is the strongest genetic risk factor for $\mathrm{AD}$ [162]. Reduction in
A $\beta$ was observed in APP duplication COs treated with APOE3-carrying microglia-like cells compared to the treatment with APOE4-carring microglia-like cells. This indicates that the APOE4 variant hinders the ability of microglia to clear $\mathrm{A} \beta$ in $\mathrm{AD}$ [78]. Despite high levels of astrocytes by six months, APOE4 COs still have high levels of $A \beta$ and significantly lower levels of full-length APOE protein, indicating impaired $A \beta$ clearance by astrocytes compared to APOE3 COs [78]. APOE4 COs also exhibit increased $A \beta$ aggregates and pTau (S202/T205) levels compared to APOE3 COs. Although AD-like pathology is delayed in APOE4 COs (six months) compared to FAD COs (two months) [43], these results illustrate that the APOE4 variant alone is sufficient to make the COs vulnerable to AD-like pathology [78].

To further understand and observe if APOE4 effects can be reversed, hiPSCs with the APOE4 allele were gene edited to APOE3 via CRISPR/Cas9 and used to generate COs. The gene-edited COs showed a non-significant decrease in $A \beta 42, A \beta 40$ levels and early endosomes possibly pointing toward additional factors in the hiPSC line that may be responsible for AD-like pathology [78]. Higher APOE levels correlated with an increase in $A \beta$ uptake by astrocytes and microglia [78]. Similar to $2 D$ neurons, $A \beta$ accumulation was reduced in APOE3 (CRISPR/Cas9 edited) organoids compared to APOE4 COs, indicating that AD-like pathology can be reversed by gene editing APOE4 to APOE3 [78].

Although APOE4 COs are more susceptible to AD-like pathology, the affected neuronal subpopulations have been explored only in 2D cultured neurons [163]. Cholinergic neurons derived from AD patients with the APOE3/4 genotype (AD-E3/E4), FAD and control hiPSCs are all electrophysiologically active [163]. However, compared to control neurons, AD-E3/E4 neurons show a significant increase in the ratio of $A \beta 42 / A \beta 40$ and altered responses to $\gamma$-secretase inhibitors. When exposed to low levels of ionomycin (calcium ionophore), AD-E3/E4 neurons show a 50\% reduction in viability compared to controls [163]. ADE3/E4 neurons also display increased cytoplasmic calcium and excitotoxic death [163], indicating that these neurons are more susceptible to glutamate induced excitotoxicity, which is consistent with the increased risk of $\mathrm{AD}$ associated with the E4 allele [162].

Another study analyzed neurons derived from hiPSCs with APOE3/3 and APOE4/4 genotypes [164]. APOE4/4 neurons produced more fragmented APOE with higher $\mathrm{A} \beta$ and $\mathrm{pTau}$ levels (S396/S404, S202/T205) compared to APOE3/3 neurons [164]. The APOE4/4 genotype affected only GABAergic neurons, making them more susceptible to degeneration compared to glutamatergic and dopaminergic neurons [164]. When the APOE4/4 line was genetically edited to APOE3/3 or treated with a structure corrector (such as PH002), the neurons showed higher levels of full-length APOE with decreased $A \beta$ and pTau levels [164], rescuing AD-like phenotypes. 
Interestingly, $\mathrm{A} \beta$ levels did not change in APOE knockout (KO) neurons treated with purified recombinant APOE3 or APOE4, indicating that $A \beta$ aggregation is dependent on endogenous levels of APOE [164]. Human astrocyte-derived APOE4 also does not alter $A \beta$ and pTau levels in APOE $\mathrm{KO}$ neurons, indicating that the gain of toxic effects is probably neuron specific [164]. More investigations are required to assess the selective neuronal vulnerability to different $A \beta$ and tau species in AD.

Neurons derived from hiPSCs with the FAD APP London variant showed different APP processing depending on the differentiation fate of these neurons [165]. For example, an increase in pathogenic $A \beta 42$ production in rostrally directed neurons compared to caudally directed neurons has been observed. In addition, the $\alpha$ - and $\beta$-secretases and Anterior Pharynx Defective 1 , a component of $\gamma$-secretase, were differentially expressed between different neuronal fates thereby differentially affecting APP processing [165]. The pTau (S202/T205) and total tau levels were also elevated in rostral neurons compared to neurons directed to caudal fates [165]. Control neurons without a FAD variant exposed to soluble AD-brain extracts also show increased tau phosphorylation in rostrally directed neurons, while tau phosphorylation remains unaffected in neurons directed to caudal fates [165]. This model illustrates that neuronal subtypes in different brain regions respond differently to the APP variant in terms of APP processing and tau homeostasis $[165,166]$, which may partially dictate the pattern of selective regional and neuronal cell vulnerability in human $\mathrm{AD}$.

In another study, accumulation of tau in neurons led to increased vulnerability to oxidative stress. Cortical neurons derived from FTD patients with the MAPT heterozygous variant A152T showed time-dependent increases in pTau levels (S202/T205, T231/S235, S396/S404, S396) and decreases in the solubility of tau protein [94]. These neurons also showed an increase in ubiquitinated proteins and markers of autophagy, indicating a disruption in protein degradation and clearance via Ubiquitin Proteasome System and the autophagylysosome-system [94]. Treatments of A152T neurons with different compounds (mitochondrial electron transport chain inhibitors, excitotoxins, or proteotoxins) showed selective neuronal loss [94], suggesting that pTau accumulation in A152T neurons is associated with increased stress vulnerability. Using CRISPR/Cas9 genomic engineering, A152T neurons were modified to disrupt the MAPT gene resulting in a downregulation of tau expression [94]. When these neurons lacking MAPT were treated with the same set of compounds, there was a rescue of stress vulnerability with an increase in viability similar to healthy control neurons [94]. This model demonstrates that accumulation of tau makes neurons more vulnerable to stress leading to neurodegeneration. Different factors such as APOE genotype variants and drug treatment should be studied in both 2D and 3D cultures, especially COs, to further understand the selective neuronal/regional vulnerability associated with $\mathrm{AD}$.

\section{Neural circuits}

COs lack proper spatial information important for establishing functional neural circuits as observed in vivo [16]. Fusion of regionally specified COs would provide a more physiologically relevant platform to study neuronal circuits. For example, the dorsal/ventral forebrainspheroid model creates a gradient of trophic factors that encourages interneuron migration, resulting in diverse and complex $3 \mathrm{D}$ brain regions with functional neuronal networks [113].

Medial ganglionic eminence organoids (MGEOs) and COs have been fused to generate functional neural networks to study interneuron migration [96]. Individual neurons in COs and MGEOs showed calcium surges resulting in synchronized neuronal activity [96]. Following treatment with bicuculline, a GABA receptor antagonist, these assembloids show an increase in calcium surge synchronization [96]. This indicates that GABAergic inhibition increases neural activity in both COs and MGEOs [96].

Human cortical spheroids (hCS) and subpallium spheroids (hSS) have also been fused to study interneuron migration, and changes in electrical activity have been found before and after the fusion [113]. Neurons that migrated from the hSS to the hCS display a doubled maximum action potential generation rate compared to non-migrated cells and neurons in unfused hSS [113]. Whole-cell voltage clamp recordings of migrated neurons also display excitatory and inhibitory postsynaptic currents (EPSCs and IPSCs) [113]. However, these neurons receive more EPSCs than IPSCs with a 3-fold increase in synaptic input. EPSCs have also been observed upon electrical simulation indicating the establishment of a functional neural network after assembly of hCS and hSS [113].

Alternative models have been established that fuse regionally specified COs or use distinct classes of neurons within a microfluidics compartment to reconstruct neuronal circuitry $[16,167]$. For example, in vitro models that reconstruct cortico-striatal and cortico-cortico circuits have been developed to understand molecular mechanisms underlying Huntington's disease and ASD, respectively [16, 167]. In some cases, MEA has been utilized to assess localized electrical activities within these models. However, a current challenge relates to correlating acquired electrical activities with the identity of neuronal subtypes. Advances in genetically encoded calcium and voltage indicators $[168,169]$ can potentially provide a solution for correlating electrical activities acquired with MEA with distinct neuronal subpopulations.

Improvements in design and culturing protocol can also aid in establishing mature neural circuits. An example is the use of fetal brain-derived ECM, rich in chondroitin sulfate, hyaluronan and heparin sulfate proteoglycan, which contain 
important differentiation/developmental cues [170]. This matrix provides better neuronal and astrocyte maturation and sustained oscillatory activity. Compared to controls, fetal ECM constructs show higher electrically active neuronal populations and spontaneous electrical activity. The ECM constructs also show coordinated physiological activity within each cluster of cells with highly correlated characteristics [170].

\section{Biomarker identification and drug screening}

Compared to 2D cultures, COs reproduce human conditions like AD more closely [35, 36, 45, 138]. Single cell transcriptomics of patient derived COs have shown considerable promise in identifying novel biomarker candidates $[15,132,171-173]$. $\beta$ and $\gamma$-secretase inhibitors decrease $\mathrm{A} \beta$ and pTau accumulation in COs $[35,36,45,138]$. Treatments with other compounds like heparin, heparinase III, DAPT [81], IL-4 [41] and nicotinamide mononucleotide [75] also show a reduction in $\mathrm{A} \beta$ induced toxicity and reversal of other $\mathrm{AD}$-like pathologies such as pTau and A $\beta$ plaque-like accumulation. Further studies are required to investigate if treatment and removal of toxic $A \beta$ or tau species prior to the onset of toxic protein aggregation, altered synaptic function and cognitive deficits, can prevent, and/or delay the development of AD-like pathology in COs.

COs also provide greater translatability to clinical trials and thus accelerate the drug discovery process. These trials either target clearance of $A \beta$ and tau or focus on immunotherapy involving active or passive immunization. Several of them have failed in Phase 2/3 clinical trials due to lack of efficacy or toxicity issues [29-34, 174-177]. The development of a human choroid plexus organoid [178] may accelerate the drug discovery process since it is involved in the production of CSF which regulates movement of nutrients/drugs into the brain [179]. Strategies could be devised to employ choroid plexus organoids in high-throughput testing of all FDA-approved drugs for their ability to provide therapeutic benefit in AD. Based on bioavailability, safety, efficacy, and clearance of these drugs in the organoids, they can potentially be quickly repurposed as $\mathrm{AD}$ therapeutics or prescribed for off-label use in treating $\mathrm{AD}$.

Although most of the current $\mathrm{CO}$ models are based on FAD variants (Table 1), it is also important to use the $\mathrm{CO}$ platform to model $\mathrm{AD}$-associated risk factors such as diabetes, hypertension, ischemia, TBI, smoking, and other environmental factors by modifying culture conditions [180]. For example, exposure of COs to heme results in brain injury leading to extensive structural disorganization, increases in proinflammatory chemokines and neurodegeneration [181]. This can be used to model and study the mechanisms underlying TBI in COs. These novel CO models will thus help the field to understand the role of these and other risk factors in the development and progression of AD. It should be noted that CRISPR-Cas9 genomic editing has not only been used to generate isogenic controls $[45,54,75,89]$ but also used as a therapeutic strategy to modulate or revert accumulation of $A \beta$ [78]. This technology can also be used to design a high throughput screen [182] for genes associated with $A \beta$ and tau aggregation, which will shed more light on the underlying mechanisms of AD.

Funding This work was supported by the National Institutes of Health [grant number, AG056673]; the Department of Defense [grant number, W81XWH1910309]; the Alzheimer's Association [grant number, AARF-17-505009]; the Neuroscience Research Institute Pilot Award and the Chronic Brain Injury Pilot Award from The Ohio State University to H.F, M.E.H., and D.S.; and supported by Research Funds through the Abigail Wexner Research Institute at Nationwide Children's Hospital to M.E.H.

\section{Compliance with ethical standards}

Conflicts of Interest The authors declare they have no conflict of interest.

\section{References}

1. Dawson, T. M., Golde, T. E., \& Lagier-Tourenne, C. (2018). Animal models of neurodegenerative diseases. Nat Neurosci., 21(10), 1370-1379. https://doi.org/10.1038/s41593-018-0236-8.

2. Erkkinen, M. G., Kim, M.-O., \& Geschwind, M. D. (2018). Clinical Neurology and Epidemiology of the Major Neurodegenerative Diseases. Cold Spring Harbor Perspectives in Biology, 10(4). https://doi.org/10.1101/cshperspect.a033118.

3. Gan, L., Cookson, M. R., Petrucelli, L., et al. (2018). Converging pathways in neurodegeneration, from genetics to mechanisms. Nat Neurosci., 21(10), 1300-1309. https://doi.org/10.1038/ s41593-018-0237-7.Converging.

4. Fu, H., Hardy, J., \& Duff, K. E. (2018). Selective vulnerability in neurodegenerative diseases. Nat Neurosci., 21(10), 1350-1358. https://doi.org/10.1038/s41593-018-0221-2.

5. Ross, C. A., \& Poirier, M. A. (2004). Protein aggregation and neurodegenerative disease. Nat Med., 10(Suppl), S10-S17. https://doi.org/10.1038/nm1066.

6. Soto, C., \& Pritzkow, S. (2018). Protein misfolding, aggregation, and conformational strains in neurodegenerative diseases. Nat Neurosci., 21(10), 1332-1340. https://doi.org/10.1038/s41593018-0235-9.

7. Jucker, M., \& Walker, L. C. (2018). Propagation and spread of pathogenic protein assemblies in neurodegenerative diseases. Nat Neurosci., 21(10), 1341-1349. https://doi.org/10.1038/s41593018-0238-6.Propagation.

8. Centeno, E. G. Z., Cimarosti, H., \& Bithell, A. (2018). 2D versus 3D human induced pluripotent stem cell-derived cultures for neurodegenerative disease modelling. Mol Neurodegener., 13(1), 27. https://doi.org/10.1186/s13024-018-0258-4.

9. Camp, J. G., Badsha, F., Florio, M., et al. (2015). Human cerebral organoids recapitulate gene expression programs of fetal neocortex development. Proc Natl Acad Sci U S A., 112(51), 1567215677. https://doi.org/10.1073/pnas.1520760112.

10. Luo, C., Lancaster, M. A., Castanon, R., Nery, J. R., Knoblich, J. A., \& Ecker, J. R. (2016). Cerebral Organoids Recapitulate 
Epigenomic Signatures of the Human Fetal Brain. Cell Rep., 17(12), 3369-3384. https://doi.org/10.1016/j.celrep.2016.12.001.

11. Lancaster, M. A., Corsini, N. S., Wolfinger, S., et al. (2017). Guided self-organization and cortical plate formation in human brain organoids. Nat Biotechnol., 35(7), 659-666. https://doi. org/10.1038/nbt.3906.

12. Sivitilli, A. A., Gosio, J. T., Ghoshal, B., et al. (2020). Robust production of uniform human cerebral organoids from pluripotent stem cells. Life Science alliance, 3(5). https://doi.org/10.26508/ 1sa.202000707.

13. Lancaster, M. A., \& Knoblich, J. A. (2014). Generation of Cerebral Organoids from Human Pluripotent Stem Cells. Nat Protoc., 9(10), 2329-2340. https://doi.org/10.1038/nprot.2014. 158.Generation.

14. Quadrato, G., Nguyen, T., Macosko, E. Z., et al. (2017). Cell diversity and network dynamics in photosensitive human brain organoids. Nature., 545(7652), 48-53. https://doi.org/10.1038/ nature22047.

15. Camp, J. G., Badsha, F., Florio, M., Kanton, S., Gerber, T., \& Wilsch-bräuninger, M. (2015). Human cerebral organoids recapitulate gene expression programs of fetal neocortex development. PNAS., 112(51), 1-6. https://doi.org/10.1073/pnas.1520760112.

16. Pasca, S. P. (2018). The rise of three-dimensional human brain cultures. Nature., 553, 437-445. https://doi.org/10.1038/ nature25032.

17. Wang, X., Tsai, J.-W., LaMonica, B., \& Kriegstein, A. R. (2011). A new subtype of progenitor cell in the mouse embryonic neocortex. Nat Neurosci., 14(5), 555-561. https://doi.org/10.1038/nn. 2807.

18. Lancaster, M. A., Renner, M., Martin, C.-A., et al. (2013). Cerebral organoids model human brain development and microcephaly. Nature., 501, 373. https://doi.org/10.1038/nature12517.

19. Hodge, R. D., Bakken, T. E., Miller, J. A., et al. (2019). Conserved cell types with divergent features in human versus mouse cortex. Nature., 573(7772), 61-68. https://doi.org/10.1038/s41586-0191506-7.

20. Di Lullo, E., \& Kriegstein, A. R. (2017). The use of brain organoids to investigate neural development and disease. Nat Rev Neurosci., 18, 573. https://doi.org/10.1038/nrn.2017.107.

21. Bhaduri, A., Andrews, M. G., Mancia Leon, W., et al. (2020). Cell stress in cortical organoids impairs molecular subtype specification. Nature., 578(7793), 142-148. https://doi.org/10.1038/ s41586-020-1962-0.

22. Tanaka, Y., Cakir, B., Xiang, Y., Sullivan, G. J., \& Park, I.-H. (2020). Synthetic Analyses of Single-Cell Transcriptomes from Multiple Brain Organoids and Fetal Brain. Cell Reports, 30(6), 1682-1689.e3. https://doi.org/10.1016/j.celrep.2020.01.038.

23. Velasco, S., Kedaigle, A. J., Simmons, S. K., et al. (2019). Individual brain organoids reproducibly form cell diversity of the human cerebral cortex. Nature., 570, 523-527. https://doi. org/10.1038/s41586-019-1289-x.

24. Qian, X., Nguyen, H. N., Song, M. M., et al. (2016). Brain Region-specific Organoids using Mini-bioreactors for Modeling ZIKV Exposure. Cell., 165(5), 1238-1254. https://doi.org/10. 1016/j.cell.2016.04.032.Brain.

25. Lanoiselee, H.-M., Nicolas, G., Wallon, D., et al. (2017). APP, PSEN1, and PSEN2 mutations in early-onset Alzheimer disease: A genetic screening study of familial and sporadic cases. PloS Med., 14(3), 1-16.

26. Haass, C., Kaether, C., Thinakaran, G., \& Sisodia, S. (2012). Trafficking and Proteolytic Processing of APP. Cold Spring Harb Perspect Med., 1-25. https://doi.org/10.1101/cshperspect. a006270.

27. Wolfe, M. S. (2020). Unraveling the complexity of $\gamma$-secretase. Semin Cell Dev Biol., 105, 3-11. https://doi.org/10.1016/j. semcdb.2020.01.005.
28. Henley, D., Raghavan, N., Sperling, R., Aisen, P., Raman, R., \& Romano, G. (2019). Preliminary Results of a Trial of Atabecestat in Preclinical Alzheimer's Disease. $N$ Engl J Med., 380(15), 1483-1485. https://doi.org/10.1056/NEJMc1813435.

29. Green, R. C., Schneider, L. S., Amato, D. A., et al. (2009). Effect of tarenflurbil on cognitive decline and activities of daily living in patients with mild Alzheimer disease: a randomized controlled trial. JAMA., 302(23), 2557-2564. https://doi.org/10.1001/jama. 2009.1866

30. Coric, V., Salloway, S., van Dyck, C. H., et al. (2015). Targeting Prodromal Alzheimer Disease With Avagacestat: A Randomized Clinical Trial. JAMA Neurol., 72(11), 1324-1333. https://doi.org/ 10.1001/jamaneurol.2015.0607.

31. Doody, R. S., Raman, R., Farlow, M., et al. (2013). A phase 3 trial of semagacestat for treatment of Alzheimer's disease. $N$ Engl $J$ Med., 369(4), 341-350. https://doi.org/10.1056/NEJMoa1210951.

32. Egan, M. F., Kost, J., Voss, T., et al. (2019). Randomized Trial of Verubecestat for Prodromal Alzheimer's Disease. N Engl J Med., 380(15), 1408-1420. https://doi.org/10.1056/NEJMoa1812840.

33. Hopkins, C. R. (2011). ACS chemical neuroscience molecule spotlight on ELND006: another $\gamma$-secretase inhibitor fails in the clinic. ACS Chem Neurosci., 2(6), 279-280. https://doi.org/10. $1021 / \mathrm{cn} 2000469$.

34. Vormfelde, S, V., Pezous, N., Lefèvre, G., et al. A Pooled Analysis of Three Randomized Phase I/IIa Clinical Trials Confirms Absence of a Clinically Relevant Effect on the QTc Interval by Umibecestat. Clinical and Translational Science. https://doi.org/10.1111/cts.12832

35. Choi, S. H., Kim, Y. H., Hebisch, M., et al. (2014). A threedimensional human neural cell culture model of Alzheimer's disease. Nature., 515(7526), 274-278. https://doi.org/10.1038/ nature 13800.

36. Kim, Y. H., Choi, S. H., Avanzo, C. D., et al. (2015). A 3D human neural cell culture system for modeling Alzheimer' $\mathrm{s}$ disease. Nature Protocols, 10(7). https://doi.org/10.1038/nprot.2015.065.

37. Kwak, S. S., Washicosky, K. J., Brand, E., et al. (2020). Amyloid$\beta 42 / 40$ ratio drives tau pathology in $3 \mathrm{D}$ human neural cell culture models of Alzheimer's disease. Nat Commun., 11(1377), 1-14 https://ghr.nlm.nih.gov/condition/alzheimer-disease\#.

38. Wong, H.-K., Sakurai, T., Oyama, F., et al. (2005). beta Subunits of voltage-gated sodium channels are novel substrates of beta-site amyloid precursor protein-cleaving enzyme (BACE1) and gamma-secretase. J Biol Chem., 280(24), 23009-23017. https://doi. org/10.1074/jbc.M414648200.

39. Esler, W. P., Kimberly, W. T., Ostaszewski, B. L., et al. (2002). Activity-dependent isolation of the presenilin- gamma -secretase complex reveals nicastrin and a gamma substrate. Proc Natl Acad Sci U S A., 99(5), 2720-2725. https://doi.org/10.1073/pnas. 052436599.

40. Cheret, C., Willem, M., Fricker, F. R., et al. (2013). Bace1 and Neuregulin-1 cooperate to control formation and maintenance of muscle spindles. EMBO J., 32(14), 2015-2028. https://doi.org/10. 1038/emboj.2013.146.

41. Papadimitriou, C., Celikkaya, H., Cosacak, M. I., et al. (2018). 3D Culture Method for Alzheimer's Disease Modeling Reveals Interleukin-4 Rescues A b 42-Induced Loss of Human Neural Stem Cell Plasticity Article 3D Culture Method for Alzheimer's Disease Modeling Reveals Interleukin-4 Rescues A b 42-Induced Loss of Hum. Developmental Cell, 46(1), 85-101.e8. https://doi. org/10.1016/j.devcel.2018.06.005.

42. Rouleau, N., Cantley, W. L., Liaudanskaya, V., et al. (2020). A Long-Living Bioengineered Neural Tissue Platform to Study Neurodegeneration. Macromol Biosci., 20, 1-8. https://doi.org/ 10.1002/mabi.202000004.

43. Raja, W. K., Mungenast, A. E., Lin, Y., et al. (2016). SelfOrganizing 3D Human Neural Tissue Derived from Induced 
Pluripotent Stem Cells Recapitulate Alzheimer's Disease Phenotypes. PLoS One., 1-18. https://doi.org/10.1371/journal. pone.0161969.

44. Yin, J., VanDongen, A, M. Enhanced Neuronal Activity and Asynchronous Calcium Transients Revealed in a 3D Organoid Model of Alzheimer's Disease. 2020. https://doi.org/10.1101/ 2020.07.07.192781. bioRxiv preprint

45. Alić, I., Goh, P.A., Murray, A. et al. (2020). Patient-specific Alzheimer-like pathology in trisomy 21 cerebral organoids reveals BACE2 as a gene dose-sensitive AD suppressor in human brain. Mol Psychiatry. https://doi.org/10.1038/s41380-020-08065.

46. Gonzalez, C., Armijo, E., Bravo-alegria, J., Mays, A. B. C. E., \& Soto, C. (2018). Modeling amyloid beta and tau pathology in human cerebral organoids. Mol Psychiatry., 23, 2363-2374. https://doi.org/10.1038/s41380-018-0229-8.

47. Lott, I. T., \& Head, E. (2019). Dementia in Down syndrome: unique insights for Alzheimer disease research. Nat Rev Neurol., 15(614), 135-147.

48. Pavoni, S., Jarray, R., Nassor, F., et al. (2018). Small-molecule induction of $A \beta-42$ peptide production in human cerebral organoids to model Alzheimer' $\mathrm{s}$ disease associated phenotypes. PLoS One., 1-15.

49. Choi, H., Jun, H., Jinhee, K., et al. (2019). Acetylation changes tau interactome to degrade tau in Alzheimer' $\mathrm{s}$ disease animal and organoid models. Aging Cell, (March), 1-13. https://doi.org/10. 1111/acel.13081.

50. Zhang, L. (2013). The role of HDAC6 in Alzheimer's disease. $J$ Alzheimers Dis., 33(2), 283-295. https://doi.org/10.3233/JAD2012-120727.

51. Nassor, F., Jarray, R., Biard, D. S. F., et al. (2020). Long Term Gene Expression in Human Induced Pluripotent Stem Cells and Cerebral Organoids to Model a Neurodegenerative Disease. Front Cell Neurosci., 14(February), 1-7. https://doi.org/10.3389/fncel. 2020.00014.

52. Honson, N. S., \& Kuret, J. (2008). Tau aggregation and toxicity in tauopathic neurodegenerative diseases. J Alzheimers Dis., 14(4), 417-422. https://doi.org/10.3233/jad-2008-14409.

53. Liu, F., \& Gong, C.-X. (2008). Tau exon 10 alternative splicing and tauopathies. Mol Neurodegener., 3(1), 8. https://doi.org/10. 1186/1750-1326-3-8.

54. Imamura, K., Sahara, N., Kanaan, N. M., et al. (2016). Calcium dysregulation contributes to neurodegeneration in FTLD patient iPSC-derived neurons. Sci Rep., 6, 1-10. https://doi.org/10.1038/ srep34904

55. Medda, X., Mertens, L., Versweyveld, S., et al. (2016). Development of a Scalable, High-Throughput-Compatible Assay to Detect Tau Aggregates Using iPSC-Derived Cortical Neurons Maintained in a Three-Dimensional Culture Format. $J$ Biomol Screen., 21(8), 804-815. https://doi.org/10.1177/ 1087057116638029.

56. Silva, M. C., Ferguson, F. M., Cai, Q., et al. (2019). Targeted degradation of aberrant tau in frontotemporal dementia patientderived neuronal cell models. eLife, 8:e45457, 1-8:e45431. https://doi.org/10.7554/eLife.45457.

57. Miguel, L., Rovelet-Lecrux, A., Feyeux, M., et al. (2019). Detection of all adult Tau isoforms in a 3D culture model of iPSC-derived neurons. Stem Cell Res., 40, 101541. https://doi. org/10.1016/j.scr.2019.101541.

58. Nakamura, M., Shiozawa, S., Tsuboi, D., et al. (2019). Pathological Progression Induced by the Frontotemporal Dementia-Associated R406W Tau Mutation in Patient-Derived iPSCs. Stem cell reports., 13(4), 684-699. https://doi.org/10. 1016/j.stemcr.2019.08.011.

59. Seo, J., Kritskiy, O., Watson, L. A., et al. (2017). Inhibition of p25/ Cdk5 Attenuates Tauopathy in Mouse and iPSC Models of
Frontotemporal Dementia. J Neurosci., 37(41), 9917-9924. https://doi.org/10.1523/JNEUROSCI.0621-17.2017.

60. Lee, M., Kwon, Y.,. T., Li, M., \& Peng, J. (2000). Neurotoxicity induces cleavage of p35 to p25 by calpain. Nature, 405(5), 360 364. https://doi.org/10.1038/35012636.

61. Liu, S.-L., Wang, C., Jiang, T., Tan, L., Xing, A., \& Yu, J.T. (2016). The Role of Cdk5 in Alzheimer's Disease. Mol Neurobiol., 53(7), 4328-4342. https://doi.org/10.1007/ s12035-015-9369-x.

62. Tolosa, E., Litvan, I., Höglinger, G. U., et al. (2014). A phase 2 trial of the GSK-3 inhibitor tideglusib in progressive supranuclear palsy. Mov Disord., 29(4), 470-478. https://doi.org/10.1002/mds. 25824.

63. Höglinger, G. U., Huppertz, H.-J., Wagenpfeil, S., et al. (2014). Tideglusib reduces progression of brain atrophy in progressive supranuclear palsy in a randomized trial. Mov Disord., 29(4), 479-487. https://doi.org/10.1002/mds.25815.

64. del Ser, T., Steinwachs, K. C., Gertz, H. J., et al. (2013). Treatment of Alzheimer's disease with the GSK-3 inhibitor tideglusib: a pilot study. J Alzheimers Dis., 33(1), 205-215. https://doi.org/10.3233/ JAD-2012-120805.

65. Lovestone, S., Boada, M., Dubois, B., et al. (2015). A phase II trial of tideglusib in Alzheimer's disease. J Alzheimers Dis., 45(1), 75-88. https://doi.org/10.3233/JAD-141959.

66. Ellul, M. A., Benjamin, L., Singh, B., et al. (2020). Neurological associations of COVID-19. Lancet Neurol., 19(9), 767-783. https://doi.org/10.1016/S1474-4422(20)30221-0.

67. Ramani, A., Müller, L., Ostermann, P, N., et al. (2020). SARSCoV-2 targets neurons of 3D human brain organoids. EMBO J, 39(20):e106230. https://doi.org/10.15252/embj.2020106230.

68. Song, E., Zhang, C., Israelow, B., et al. Neuroinvasive potential of SARS-CoV-2 revealed in a human brain organoid model. 2020. https://doi.org/10.1101/2020.06.25.169946. bioRxiv preprint

69. Bagnato, S., \& Boccagni, C. (2020). Moderate/severe traumatic brain injury as a trigger of chronic neurodegeneration in humans. Neural Regen Res., 15(7), 1247-1248. https://doi.org/10.4103/ 1673-5374.272574.

70. Lai, J, D., Berlind, J, E., Fricklas, G., et al. A model of traumatic brain injury using human iPSC-derived cortical brain organoids. 2020. https://doi.org/10.1101/2020.07.05.180299. bioRxiv preprint

71. Li, N., \& Lagier-Tourenne, C. (2018). Nuclear pores: the gate to neurodegeneration. Nat Neurosci., 21(2), 156-158. https://doi. org/10.1038/s41593-017-0066-0.

72. Brettschneider, J., Del Tredici, K., Lee, V. M.-Y., \& Trojanowski, J. Q. (2015). Spreading of pathology in neurodegenerative diseases: a focus on human studies. Nat Rev Neurosci., 16(2), 109-120. https:// doi.org/10.1038/nrn3887.

73. Chen, M., Lee, H.-K., Moo, L., Hanlon, E., Stein, T., \& Xia, W. (2018). Common proteomic profiles of induced pluripotent stem cell-derived three-dimensional neurons and brain tissue from Alzheimer patients. J Proteomics., 182, 21-33. https://doi.org/ 10.1016/j.jprot.2018.04.032.

74. Brunetti, D., Torsvik, J., Dallabona, C., et al. (2016). Defective PITRM 1 mitochondrial peptidase is associated with A $\beta$ amyloidotic neurodegeneration. EMBO Mol Med., 8(3), 176-190.

75. Ivanyuk, D., Pérez, M, J., Panagiotakopoulou, V., et al. Loss of function of the mitochondrial peptidase PITRM1 induces proteotoxic stress and Alzheimer' $\mathrm{s}$ disease-like pathology in human cerebral organoids. 2020. https://doi.org/10.1101/2020.01. 27.919522. bioRxiv preprint

76. Zhu, Y., Li, C., Tao, X., et al. (2019). Nmnat restores neuronal integrity by neutralizing mutant Huntingtin aggregate-induced progressive toxicity. Proc Natl Acad Sci U S A., 116(38), 19165-19175. https://doi.org/10.1073/pnas.1904563116. 
77. Cataldo, A. M., Peterhoff, C. M., Troncoso, J. C., Gomez-isla, T., Hyman, B. T., \& Nixon, R. A. (2000). Endocytic Pathway Abnormalities Precede Amyloid Endocytic Pathway Abnormalities Precede Amyloid $\beta$ Deposition in Sporadic Alzheimer's Disease and Down Syndrome. Am J Pathol., 157(1), 277-286.

78. Lin, Y. T., Seo, J., Gao, F., et al. (2018). APOE4 causes widespread molecular and cellular alterations associated with Alzheimer's disease phenotypes in human iPSC- derived brain cell types. Neuron., 98(6), 1141-1154. https://doi.org/10.1016/j. neuron.2018.05.008.APOE4.

79. Jimenez-Orgaz, A., Kvainickas, A., Nägele, H., et al. (2018). Control of RAB7 activity and localization through the retromerTBC1D5 complex enables RAB7-dependent mitophagy. EMBO Journal, 37(2), 235-254. https://doi.org/10.15252/embj. 201797128

80. Puri, C., Vicinanza, M., \& Rubinsztein, D. C. (2018). Phagophores evolve from recycling endosomes. Autophagy., 14(8), 1475-1477. https://doi.org/10.1080/15548627.2018. 1482148 .

81. Yan, Y., Song, L., Bejoy, J., et al. (2018). Modeling Neurodegenerative Microenvironment Using Cortical Organoids Derived from Human Stem Cells. Tissue Eng Part A., 24(13-14), 1125-1137. https://doi.org/10.1089/ten.TEA.2017.0423.

82. Fan, W., Sun, Y., Shi, Z., Wang, H., \& Deng, J. (2019). Mouse induced pluripotent stem cells-derived Alzheimer's disease cerebral organoid culture and neural differentiation disorders. Neurosci Lett., 711, 134433. https://doi.org/10.1016/j.neulet. 2019.134433.

83. Park, J., Wetzel, I., Marriott, I., et al. (2018). A 3D human triculture system modeling neurodegeneration and neuroinflammation in Alzheimer's disease. Nat Neurosci., 21(7), 941-951. https://doi.org/10.1038/s41593-018-0175-4.

84. Barth, A. L., \& Ray, A. (2019). Progressive Circuit Changes during Learning and Disease. Neuron., 104(1), 37-46. https://doi.org/ 10.1016/j.neuron.2019.09.032

85. Chen, Y., Fu, A. K. Y., \& Ip, N. Y. (2019). Synaptic dysfunction in Alzheimer's disease: Mechanisms and therapeutic strategies. Pharmacol Ther., 195, 186-198. https://doi.org/10.1016/j. pharmthera.2018.11.006.

86. Jackson, J., Jambrina, E., Li, J., et al. (2019). Targeting the Synapse in Alzheimer's Disease. Front Neurosci., 13, 735. https://doi.org/10.3389/fnins.2019.00735.

87. Li, S., \& Selkoe, D. J. (2020). A mechanistic hypothesis for the impairment of synaptic plasticity by soluble $\mathrm{A} \beta$ oligomers from Alzheimer's brain. $J$ Neurochem., 154(6), 583-597. https://doi. org/10.1111/jnc. 15007.

88. Palop, J. J., \& Mucke, L. (2016). Network abnormalities and interneuron dysfunction in Alzheimer disease. Nat Rev Neurosci., 17(12), 777-792. https://doi.org/10.1038/nrn.2016.141.

89. Ghatak, S., Dolatabadi, N., Trudler, D., et al. Mechanisms of hyperexcitability in Alzheimer's disease hiPSC-derived neurons and cerebral organoids vs isogenic controls. Elife. 2019;8. https://doi. org $/ 10.7554 /$ eLife.50333

90. Sweeney, M. D., Sagare, A. P., Zlokovic, B. V., \& Angeles, L. (2018). Blood-brain barrier breakdown in Alzheimer's disease and other neurodegenerative disorders. Nat Rev Neurol., 14(3), 133-150. https://doi.org/10.1038/nrneurol.2017.188.Blood.

91. Shin, Y., Choi, S. H., Kim, E., et al. (2019). Blood - Brain Barrier Dysfunction in a 3D In Vitro Model of Alzheimer 's Disease. 3D Alzheimer's Dis Model, 1900962, 1-10. https://doi.org/10.1002/ advs.201900962.

92. Zhang, D., Pekkanen-Mattila, M., Shahsavani, M., Falk, A., Teixeira, A. I., \& Herland, A. (2014). A 3D Alzheimer's disease culture model and the induction of P21-activated kinase mediated sensing in iPSC derived neurons. Biomaterials., 35(5), 1420-1428. https://doi.org/10.1016/j.biomaterials.2013.11.028.

93. Zhao, L., Ma, Q.-L., Calon, F., et al. (2005). Role of p21-activated kinase pathway defects in the cognitive deficits of Alzheimer disease. Nat Neurosci., 9(2), 234-242. https://doi.org/10.1038/ nn1630.

94. Silva, M. C., Cheng, C., Mair, W., et al. (2016). Human iPSCDerived Neuronal Model of Tau-A152T Frontotemporal Dementia Reveals Tau-Mediated Mechanisms of Neuronal Vulnerability. Stem Cell Reports., 7(3), 325-340. https://doi.org/ 10.1016/j.stemcr.2016.08.001.

95. Quadrato, G., Nguyen, T., Macosko, E. Z., et al. (2017). Cell Diversity and Network Dynamics in Photosensitive Human Brain Organoids. Nature., 545(7652), 48-53. https://doi.org/10. 1038/nature22047.Cell.

96. Xiang, Y., Tanaka, Y., Patterson, B., et al. (2017). Fusion of Regionally Specified hPSC-Derived Organoids Models Human Brain Development and Interneuron Migration. Cell Stem Cell, 21(3), 383-398.e7. https://doi.org/10.1016/j.stem.2017.07.007.

97. Miller, J. D., Ganat, Y. M., Kishinevsky, S., et al. (2013). Human iPSC-based modeling of late-onset disease via progerin-induced aging. Cell Stem Cell., 13(6), 691-705. https://doi.org/10.1016/j. stem.2013.11.006.

98. Janssens, S., Schotsaert, M., Karnik, R., et al. (2018). Zika Virus Alters DNA Methylation of Neural Genes in an Organoid Model of the Developing Human Brain. mSystems, 3(1). https://doi.org/ 10.1128/mSystems.00219-17.

99. Soto, C., \& Pritzkow, S. (2018). Protein misfolding, aggregation, and conformational strains in neurodegenerative diseases. Nat Neurosci., 21(10), 1332-1340. https://doi.org/10.1038/s41593018-0235-9.Protein.

100. Pamies, D., Block, K., Lau, P., et al. (2018). Rotenone exerts developmental neurotoxicity in a human brain spheroid model. Toxicol Appl Pharmacol., 354, 101-114. https://doi.org/10.1016/ j.taap.2018.02.003.Rotenone.

101. Zambuto, S. G., Serrano, J. F., Vilbert, A. C., Lu, Y., \& Harley, B. A. C. (2019). Pedron S. Response of neuroglia to hypoxia-induced oxidative stress using enzymatically crosslinked hydrogels., 217, 1-27. https://doi.org/10.1101/799692.

102. Vera, E., Bosco, N., \& Studer, L. (2016). Generating late-onset human IPSC-based disease models by inducing neuronal agerelated phenotypes through telomerase manipulation. Cell Rep., 17(4), 1184-1192. https://doi.org/10.1016/j.celrep.2016.09.062. GENERATING.

103. Ahmed, M. S., Ikram, S., Bibi, N., \& Mir, A. (2018). HutchinsonGilford Progeria Syndrome: A Premature Aging Disease. Mol Neurobiol., 55, 4417-4427. https://doi.org/10.1007/s12035-0170610-7.

104. Ormel, P. R., De Sá, R. V., Van Bodegraven, E. J., et al. (2018). Microglia innately develop within cerebral organoids. Nat Commun., 9(4167), 1-14. https://doi.org/10.1038/s41467-01806684-2.

105. Sloan, S. A., Darmanis, S., Huber, N., et al. (2017). Human Astrocyte Maturation Captured in 3D Cerebral Cortical Spheroids Derived from Pluripotent Stem Cells. Neuron., 95, 779-790. https://doi.org/10.1016/j.neuron.2017.07.035.

106. McQuade, A., Coburn, M., Tu, C. H., Hasselmann, J., Davtyan, H., \& Blurton-Jones, M. (2018). Development and validation of a simplified method to generate human microglia from pluripotent stem cells. Mol Neurodegener., 13(1), 67. https://doi.org/10.1186/ s13024-018-0297-x.

107. Schmunk, G., Kim, CN., Soliman, S, S., et al. Human microglia upregulate cytokine signatures and accelerate maturation of neural networks. 2020. https://doi.org/10.1101/2020.03.24.006874

108. Brüll, M., Spreng, A., Gutbier, S., et al. (2020). Incorporation of Stem Cell-Derived Astrocytes into Neuronal Organoids to Allow 
Neuro-Glial Interactions in Toxicological Studies. ALTEX - Altern to Anim Exp, 37, 1-20. https://doi.org/10.14573/altex.1911111.

109. Marton, R. M., Miura, Y., Sloan, S. A., et al. (2019). Differentiation and maturation of oligodendrocytes in human three-dimensional neural cultures. Nat Neurosci., 22(3), 484-491. https://doi.org/10. 1038/s41593-018-0316-9.

110. Madhavan, M., Nevin, Z. S., Shick, H. E., et al. (2018). Induction of myelinating oligodendrocytes in human cortical spheroids. Nat Methods., 15(9), 700-706. https://doi.org/10.1038/s41592-0180081-4.

111. Lancaster, M. A., \& Knoblich, J. A. (2014). Generation of cerebral organoids from human pluripotent stem cells. Nat Protoc., 9(10), 2329-2340. https://doi.org/10.1038/nprot.2014.158.

112. Cai, H., Ao, Z., Hu, L., et al. Acoustofluidic Assembly of 3D Neurospheroids to Model Alzheimer's Disease. 2020. https://doi. org/10.1101/2020.03.03.972299

113. Birey, F., Andersen, J., Makinson, C. D., et al. (2017). Assembly of functionally integrated human forebrain spheroids. Nature., 545(7652), 54-59. https://doi.org/10.1038/nature22330. Assembly.

114. Monzel, A. S., Smits, L. M., Hemmer, K., et al. (2017). Derivation of Human Midbrain-Specific Organoids from Neuroepithelial Stem Cells. Stem cell reports., 8(5), 1144-1154. https://doi.org/ 10.1016/j.stemcr.2017.03.010

115. Berger, E., Magliaro, C., Paczia, N., et al. (2018). Millifluidic culture improves human midbrain organoid vitality and differentiation. Lab Chip., 18(3172), 3172-3183. https://doi.org/10.1039/ c81c00206a.

116. Qian, X., Jacob, F., Song, M. M., Nguyen, H. N., Song, H., \& Ming, G.-L. (2018). Generation of human brain region-specific organoids using a miniaturized spinning bioreactor. Nat Protoc., 13(3), 565-580. https://doi.org/10.1038/nprot.2017.152.

117. Pham, M. T., Pollock, K. M., Rose, M. D., et al. (2018). Generation of human vascularized brain organoids. Neuroreport., 29(7), 588-593. https://doi.org/10.1097/WNR. 0000000000001014.

118. Ferdous, A., Caprioli, A., Iacovino, M., et al. (2009). Nkx2-5 transactivates the Ets-related protein 71 gene and specifies an endothelial/endocardial fate in the developing embryo. Proc Natl Acad Sci U S A., 106(3), 814-819. https://doi.org/10.1073/pnas. 0807583106.

119. Cakir, B., Xiang, Y., Tanaka, Y., et al. (2019). Engineering of human brain organoids with a functional vascular-like system. Nat Methods., 16(11), 1169-1175. https://doi.org/10.1038/ s41592-019-0586-5.

120. Mansour, A. A., Gonçalves, J. T., Bloyd, C. W., et al. (2018). An in vivo model of functional and vascularized human brain organoids. Nat Biotechnol., 36(5), 432-441. https://doi.org/10. $1038 /$ nbt.4127.

121. Li, Y., Sun, X., Liu, H., et al. (2019). Development of Human in vitro Brain-blood Barrier Model from Induced Pluripotent Stem Cell-derived Endothelial Cells to Predict the in vivo Permeability of Drugs. Neurosci Bull., 35(6), 996-1010. https:// doi.org/10.1007/s12264-019-00384-7.

122. Wang, Y. I., Abaci, H. E., \& Shuler, M. L. (2017). Microfluidic blood-brain barrier model provides in vivo-like barrier properties for drug permeability screening. Biotechnol Bioeng., 114(1), 184 194. https://doi.org/10.1002/bit.26045.

123. Stebbins, M. J., Gastfriend, B. D., Canfield, S. G., et al. (2019). Human pluripotent stem cell - derived brain pericyte - like cells induce blood-brain barrier properties. Sci Adv., 5, 1-16.

124. Campisi, M., Shin, Y., Osaki, T., Hajal, C., Chiono, V., \& Kamm, R. D. (2018). 3D self-organized microvascular model of the human blood-brain barrier with endothelial cells, pericytes and astrocytes. Biomaterials., 180, 117-129. https://doi.org/10.1016/j. biomaterials.2018.07.014
125. Canfield, S. G., Stebbins, M. J., Morales, B. S., et al. (2017). An isogenic blood-brain barrier model comprising brain endothelial cells, astrocytes, and neurons derived from human induced pluripotent stem cells. J Neurochem., 140(6), 874-888. https://doi.org/ 10.1111/jnc. 13923.

126. Vatine, G. D., Barrile, R., Workman, M. J., et al. (2019). Human iPSC-Derived Blood-Brain Barrier Chips Enable Disease Modeling and Personalized Medicine Applications. Cell Stem Cell, 24(6), 995-1005.e6. https://doi.org/10.1016/j.stem.2019.05. 011.

127. Trujillo, C. A., Gao, R., Negraes, P. D., et al. (2019). Complex Oscillatory Waves Emerging from Cortical Organoids Model Early Human Brain Network Development. Cell Stem Cell, 25(4), 558-569.e7. https://doi.org/10.1016/j.stem.2019.08.002.

128. Meyer, K., Feldman, H. M., Lu, T., et al. (2019). REST and Neural Gene Network Dysregulation in iPSC Models of Alzheimer's Disease. Cell Reports, 26(5), 1112-1127.e9. https://doi.org/10. 1016/j.celrep.2019.01.023.

129. Kanton, S., Boyle, M. J., He, Z., et al. (2019). Organoid single-cell genomic atlas uncovers human-specific features of brain development. Nature., 574, 418-421.

130. Mathys, H., Davila-Velderrain, J., Peng, Z., et al. (2019). Singlecell transcriptomic analysis of Alzheimer's disease. Nature., 570(7761), 332-337. https://doi.org/10.1038/s41586-019-1195-2.

131. Kathuria, A., Lopez-Lengowski, K., Vater, M., McPhie, D., Cohen, B. M., \& Karmacharya, R. (2020). Transcriptome analysis and functional characterization of cerebral organoids in bipolar disorder. Genome Med., 12(1), 34. https://doi.org/10.1186/ s13073-020-00733-6.

132. Kathuria, A., Lopez-Lengowski, K., Jagtap, S. S., et al. (2020). Transcriptomic Landscape and Functional Characterization of Induced Pluripotent Stem Cell-Derived Cerebral Organoids in Schizophrenia. JAMA psychiatry., 77(7), 745-754. https://doi. org/10.1001/jamapsychiatry.2020.0196.

133. Wang, P., Mokhtari, R., Pedrosa, E., et al. (2017). CRISPR/Cas9mediated heterozygous knockout of the autism gene CHD8 and characterization of its transcriptional networks in cerebral organoids derived from iPS cells. Mol Autism., 8, 11. https://doi. org/10.1186/s13229-017-0124-1.

134. Burgess, D. J. (2019). Spatial transcriptomics coming of age. Nat Rev Genet., 20, 317. https://doi.org/10.1038/s41576-019-0129-z.

135. Chen, W., Lu, A., Craessaerts, K., Pavie, B., Frigerio, C, S.. Spatial and temporal transcriptomics reveal microglia-astroglia crosstalk in the amyloid- $\beta$ plaque cell niche of Alzheimer's disease. 2019. https://doi.org/10.1101/719930

136. Hondius, D. C., van Nierop, P., Li, K. W., et al. (2016). Profiling the human hippocampal proteome at all pathologic stages of Alzheimer's disease. Alzheimers Dement., 12(6), 654-668. https://doi.org/10.1016/j.jalz.2015.11.002.

137. Lachén-Montes, M., González-Morales, A., Zelaya, M. V., et al. (2017). Olfactory bulb neuroproteomics reveals a chronological perturbation of survival routes and a disruption of prohibitin complex during Alzheimer's disease progression. Sci Rep., 7(1), 9115. https://doi.org/10.1038/s41598-017-09481-x.

138. Lee, H.-K., Velazquez Sanchez, C., Chen, M., et al. (2016). Three Dimensional Human Neuro-Spheroid Model of Alzheimer's Disease Based on Differentiated Induced Pluripotent Stem Cells. PLoS One., 11(9), e0163072. https://doi.org/10.1371/journal. pone. 0163072 .

139. Mcclure-begley, T, D., Ebmeier, C, C., Ball, K, E., Jacobsen, J, R. Cerebral organoid proteomics reveals signatures of dysregulated cortical development associated with human trisomy 21. 2018. https://doi.org/10.1101/315317

140. Bouso, J. C., Palhano-fontes, F., Rodríguez-fornells, A., et al. (2015). Long-term use of psychedelic drugs is associated with differences in brain structure and personality in humans \$. Eur 
Neuropsychopharmacol., 25(4), 483-492. https://doi.org/10. 1016/j.euroneuro.2015.01.008.

141. Dakic, V., Minardi Nascimento, J., Costa Sartore, R., et al. (2017). Short term changes in the proteome of human cerebral organoids induced by 5-MeO-DMT. Sci Rep., 7(1), 12863. https://doi.org/ 10.1038/s41598-017-12779-5.

142. Fiehn, O. (2002). Metabolomics-the link between genotypes and phenotypes. Plant Mol Biol., 48(1-2), 155-171.

143. Johnson, C. H., Ivanisevic, J., \& Siuzdak, G. (2016). Metabolomics: beyond biomarkers and towards mechanisms. Nat Rev Mol Cell Biol., 17(7), 451-459. https://doi.org/10.1038/ nrm.2016.25.Metabolomics.

144. Patti, G. J., Yanes, O., \& Siuzdak, G. (2012). Innovation: Metabolomics: the apogee of the omics trilogy. Nat Rev Mol Cell Biol., 13(4), 263-269. https://doi.org/10.1038/nrm3314.

145. Ibanez, C., Simó, C., Barupal, D. K., et al. (2013). A new metabolomic workflow for early detection of Alzheimer's disease. J Chromatogr A., 1302, 65-71.

146. Casanova, R., Varma, S., Simpson, B., et al. (2016). Blood metabolite markers of preclinical Alzheimer's disease in two longitudinally followed cohorts of older individuals. Alzheimer's Dement., 12(7), 815-822. https://doi.org/10.1016/j.jalz.2015.12. 008 .

147. González-Domínguez, R., García-Barrera, T., \& Gómez-Ariza, J. L. (2014). Metabolomic study of lipids in serum for biomarker discovery in Alzheimer's disease using direct infusion mass spectrometry. J Pharm Biomed Anal., 98, 321-326. https://doi.org/10. 1016/j.jpba.2014.05.023.

148. Kaddurah-Daouk, R., Rozen, S., Matson, W., et al. (2011). Metabolomic changes in autopsy-confirmed Alzheimer's disease. Alzheimers Dement., 7(3), 309-317. https://doi.org/10.1016/j.jalz. 2010.06.001.

149. Peña-Bautista, C., Roca, M., Hervás, D., et al. (2019). Plasma metabolomics in early Alzheimer's disease patients diagnosed with amyloid biomarker. J Proteomics., 200, 144-152. https:// doi.org/10.1016/j.jprot.2019.04.008.

150. Varma, V. R., Oommen, A. M., Varma, S., et al. (2018). Brain and blood metabolite signatures of pathology and progression in Alzheimer disease: A targeted metabolomics study. PLoS Med., 15(1), e1002482. https://doi.org/10.1371/journal.pmed.1002482.

151. Gomez-Giro, G., Arias-Fuenzalida, J., Jarazo, J., et al. (2019). Synapse alterations precede neuronal damage and storage pathology in a human cerebral organoid model of CLN3-juvenile neuronal ceroid lipofuscinosis. Acta Neuropathol Commun., 7(1), 222. https://doi.org/10.1186/s40478-019-0871-7.

152. Stopschinski, B. E., \& Diamond, M. I. (2017). The prion model for progression and diversity of neurodegenerative diseases. Lancet Neurol., 16(4), 323-332. https://doi.org/10.1016/S14744422(17)30037-6.

153. Groveman, B. R., Foliaki, S. T., Orru, C. D., et al. (2019). Sporadic Creutzfeldt-Jakob disease prion infection of human cerebral organoids. Acta Neuropathol Commun., 7(12), 1-12.

154. Reilly, P., Winston, C. N., Baron, K., Trejo, M., \& Edward, M. (2017). Novel human neuronal tau model exhibiting neurofibrillary tangles and transcellular propagation. Neurobiol Dis., 106, 222-234. https://doi.org/10.1016/j.nbd.2017.06.005.Novel.

155. Chen, X.-Q., \& Mobley, W. C. (2019). Alzheimer Disease Pathogenesis: Insights From Molecular and Cellular Biology Studies of Oligomeric A $\beta$ and Tau Species. Front Neurosci., 13, 659. https://doi.org/10.3389/fnins.2019.00659.

156. Usenovic, M., Niroomand, S., Drolet, R. E., et al. (2015). Internalized Tau Oligomers Cause Neurodegeneration by Inducing Accumulation of Pathogenic Tau in Human Neurons Derived from Induced Pluripotent Stem Cells. J Neurosci., 35(42), 14234-14250. https://doi.org/10.1523/JNEUROSCI. 1523-15.2015.
157. Dai, C.-L., Hu, W., Tung, Y. C., Liu, F., Gong, C.-X., \& Iqbal, K. (2018). Tau passive immunization blocks seeding and spread of Alzheimer hyperphosphorylated Tau-induced pathology in $3 \times$ Tg-AD mice. Alzheimers Res Ther., 10(1), 13. https://doi.org/10. 1186/s13195-018-0341-7.

158. Lasagna-Reeves, C. A., Castillo-Carranza, D. L., Sengupta, U., et al. (2012). Alzheimer brain-derived tau oligomers propagate pathology from endogenous tau. Sci Rep., 2, 700. https://doi.org/ 10.1038/srep00700.

159. Tian, H., Davidowitz, E., Lopez, P., Emadi, S., Moe, J., \& Sierks, M. (2013). Trimeric tau is toxic to human neuronal cells at low nanomolar concentrations. Int J Cell Biol., 2013. https://doi.org/ $10.1155 / 2013 / 260787$.

160. Sinha, M. S., Schultz, A. A., Civitelli, L., et al. (2018). Alzheimer's disease pathology propagation by exosomes containing toxic amyloid-beta oligomers. Acta Neuropathol., 136(1), 41-56. https://doi.org/10.1007/s00401-018-1868-1.

161. Brunello, C. A., Merezhko, M., Uronen, R.-L., \& Huttunen, H. J. (2020). Mechanisms of secretion and spreading of pathological tau protein. Cell Mol Life Sci., 77(9), 1721-1744. https://doi.org/10. 1007/s00018-019-03349-1.

162. Corder, E., Saunders, A., Strittmatter, W., et al. (1993). Gene Dose of Apolipoprotein and the Risk of Alzheimer' s Disease in Late Onset Families. Science (80), 261(5123), 921-923.

163. Duan, L., Bhattacharyya, B. J., Belmadani, A., Pan, L., Miller, R. J., \& Kessler, J. A. (2014). Stem cell derived basal forebrain cholinergic neurons from Alzheimer's disease patients are more susceptible to cell death. Mol Neurodegener., 9(3), 1-14.

164. Wang, C., Najm, R., Xu, Q., et al. (2018). Gain of toxic apolipoprotein $\mathrm{E} 4$ effects in human iPSC-derived neurons is ameliorated by a small-molecule structure corrector. Nat Med., 24(5), 647-657. https://doi.org/10.1038/s41591-018-0004-z.

165. Muratore, C. R., Zhou, C., Liao, M., et al. (2017). Cell-type Dependent Alzheimer's Disease Phenotypes: Probing the Biology of Selective Neuronal Vulnerability. Stem cell reports., 9(6), 1868-1884. https://doi.org/10.1016/j.stemcr.2017.10.015.

166. Muratore, C. R., Rice, H. C., Srikanth, P., et al. (2014). The familial Alzheimer's disease APPV717I mutation alters APP processing and Tau expression in iPSC-derived neurons. Hum Mol Genet., 23(13), 3523-3536. https://doi.org/10.1093/hmg/ddu064.

167. Virlogeux, A., Moutaux, E., Christaller, W., Charlot, B., \& Bruye, J. (2018). Reconstituting Corticostriatal Network on-a-Chip Reveals the Contribution of the Presynaptic Compartment to Huntington's Disease. Cell Rep., 22, 110-122. https://doi.org/ 10.1016/j.celrep.2017.12.013.

168. Knöpfel, T., \& Song, C. (2019). Optical voltage imaging in neurons: moving from technology development to practical tool. Nat Rev Neurosci., 20(12), 719-727. https://doi.org/10.1038/s41583019-0231-4.

169. Park, J. G., \& Palmer, A. E. (2015). Properties and use of genetically encoded FRET sensors for cytosolic and organellar Ca2+ measurements. Cold Spring Harbor Protocols, 2015(1), pdb.top066043. https://doi.org/10.1101/pdb.top066043.

170. Sood, D., Cairns, D. M., Dabbi, J. M., et al. (2019). Functional maturation of human neural stem cells in a 3D bioengineered brain model enriched with fetal brain-derived matrix. Sci Rep., 9(1), 17874. https://doi.org/10.1038/s41598-019-54248-1.

171. Kathuria, A., Lopez-lengowski, K., Vater, M., Mcphie, D., \& Cohen, B. M. (2020). Transcriptome analysis and functional characterization of cerebral organoids in bipolar disorder. Genome Med., 12(34), 1-16.

172. Pollen, A. A., Nowakowski, T. J., Shuga, J., et al. (2014). Lowcoverage single-cell mRNA sequencing reveals cellular heterogeneity and activated signaling pathways in developing cerebral cortex. Nat Biotechnol., 32(10), 1053-1058. https://doi.org/10.1038/nbt.2967. 
173. Wang, P., Mokhtari, R., Pedrosa, E., et al. (2017). CRISPR/Cas9mediated heterozygous knockout of the autism gene CHD8 and characterization of its transcriptional networks in cerebral organoids derived from iPS cells. Mol Autism., 8(11), 1-17. https://doi.org/10.1186/s13229-017-0124-1.

174. Ostrowitzki, S., Lasser, R. A., Dorflinger, E., et al. (2017). A phase III randomized trial of gantenerumab in prodromal Alzheimer's disease. Alzheimers Res Ther., 9(1), 95. https://doi. org/10.1186/s13195-017-0318-y.

175. Honig, L. S., Vellas, B., Woodward, M., et al. (2018). Trial of Solanezumab for Mild Dementia Due to Alzheimer's Disease. $N$ Engl J Med., 378(4), 321-330. https://doi.org/10.1056/ NEJMoa1705971.

176. Cummings, J. L., Cohen, S., van Dyck, C. H., et al. (2018). ABBY: A phase 2 randomized trial of crenezumab in mild to moderate Alzheimer disease. Neurology., 90(21), e1889-e1897. https://doi.org/10.1212/WNL.0000000000005550.

177. Salloway, S., Sperling, R., Fox, N. C., et al. (2014). Two phase 3 trials of bapineuzumab in mild-to-moderate Alzheimer's disease. $N$ Engl J Med., 370(4), 322-333. https://doi.org/10.1056/ NEJMoa1304839.

178. Pellegrini, L., Bonfio, C., Chadwick, J., Begum, F., Skehel, M., \& Lancaster, M. A. (2020). Human CNS barrier-forming organoids with cerebrospinal fluid production. Science (80- ), 369(eaaz5626), 1-12. https://doi.org/10.1126/science.aaz5626.

179. Lun, M. P., Monuki, E. S., \& Lehtinen, M. K. (2015). Development and functions of the choroid plexus-cerebrospinal fluid system. Nat Rev Neurosci., 16(8), 445-457. https://doi.org/ 10.1038/nrn3921.

180. Edwards III, G. A., Gamez, N., Escobedo Jr., G., Calderon, O., \& Moreno-gonzalez, I. (2019). Modifiable Risk Factors for Alzheimer's Disease. Front Aging Neurosci., 11(146), 1-18. https://doi.org/10.3389/fnagi.2019.00146.

181. Harbuzariu, A., Pitts, S., Cespedes, J. C., et al. (2019). Modelling heme-mediated brain injury associated with cerebral malaria in human brain cortical organoids. Sci Rep., 9(19162), 1-14. https://doi.org/10.1038/s41598-019-55631-8.

182. Shang, W., Wang, F., Fan, G., \& Wang, H. (2017). Key elements for designing and performing a CRISPR/Cas9-based genetic screen. J Genet Genomics., 44(9), 439-449. https://doi.org/10. 1016/j.jgg.2017.09.005.

183. Moreno, E. L., Hachi, S., Hemmer, K., et al. (2015). Differentiation of neuroepithelial stem cells into functional dopaminergic neurons in 3D microfluidic cell culture. Lab Chip., 15, 2419-2428. https://doi.org/10.1039/C5LC00180C.

184. Adil, M. M., Rodrigues, G. M. C., Kulkarni, R. U., \& Rao, A. T. (2017). Efficient generation of hPSC- derived midbrain dopaminergic neurons in a fully defined, scalable, 3D biomaterial platform. Sci Rep., 7(40573), 1-11. https://doi.org/10.1038/ srep40573.

185. Monzel, A. S., Smits, L. M., Hemmer, K., et al. (2017). Derivation of Human Midbrain-Specific Organoids from Neuroepithelial Stem Cells. Stem Cell Reports., 8, 1144-1154. https://doi.org/10. 1016/j.stemcr.2017.03.010.
186. Son, M., Sim, H., Son, Y. S., et al. (2017). Distinctive genomic signature of neural and intestinal organoids from familial Parkinson's disease patient- derived induced pluripotent stem cells. Neuropathol Appl Neurobiol., 43, 584-603. https://doi.org/ 10.1111/nan.12396.

187. Jarazo, J., Barmpa, K., Rosety, I., et al. Parkinson's disease phenotypes in patient specific brain organoids are improved by HP- $\beta-\mathrm{CD}$ treatment. 2019. https://doi.org/10.1101/813089

188. Kim, H., Park, H. J., Choi, H., et al. (2019). Modeling G2019SLRRK2 Sporadic Parkinson's Disease in 3D Midbrain Organoids. Stem Cell Reports., 12(3), 518-531. https://doi.org/10.1016/j. stemcr.2019.01.020.

189. Smits, L. M., \& Reinhardt, L. (2019). Reinhardt P, et al. Modeling Parkinson's disease in midbrain-like organoids. npj Park Dis., 5, 1-8. https://doi.org/10.1038/s41531-019-0078-4.

190. Chlebanowska, P., Tejchman, A., Sułkowski, M., \& Skrzypek, K. (2020). Use of 3D Organoids as a Model to Study Idiopathic Form of Parkinson's s Disease. Int J Mol Sci., 21(694), 1-13.

191. Kwak, T. H., Hali, S., Kim, J., et al. (2020). Generation of homogeneous midbrain organoids with in vivo - like cellular composition facilitates neurotoxin-based Parkinson' s disease modeling. Stem Cells., 1-14. https://doi.org/10.1002/stem.3163.

192. Nakamura, M., Shiozawa, S., Tsuboi, D., et al. (2019). Pathological Progression Induced by the Frontotemporal Dementia- Associated R406W Tau Mutation in Patient-Derived iPSCs. Stem Cell Reports., 13, 684-699. https://doi.org/10.1016/ j.stemcr.2019.08.011.

193. Faghihi, F., Mirzaei, E., Ai, J., \& Lotfi, A. (2016). Differentiation Potential of Human Chorion-Derived Mesenchymal Stem Cells into Motor Neuron-Like Cells in Two- and Three-Dimensional Culture Systems. Mol Neurobiol., 53, 1862-1872. https://doi. org/10.1007/s12035-015-9129-y.

194. Kawada, J., Kaneda, S., Kirihara, T., et al. (2017). Generation of a Motor Nerve Organoid with Human Stem Cell-Derived Neurons. Stem Cell Reports., 9, 1441-1449. https://doi.org/10.1016/j. stemcr.2017.09.021.

195. Osaki, T., Uzel, S. G. M., \& Kamm, R. D. (2018). Microphysiological 3D model of amyotrophic lateral sclerosis ( ALS ) from human iPS-derived muscle cells and optogenetic motor neurons. Science Advances, 4(eaat5847), $1-15$.

196. Akiyama, T., Suzuki, N., Ishikawa, M., Fujimori, K., Sone, T., \& Kawada, J. (2019). Aberrant axon branching via Fos-B dysregulation in FUS -ALS motor neurons. EBioMedicine., 45, 362-378. https://doi.org/10.1016/j.ebiom.2019.06.013.

197. Conforti, P., Besusso, D., Bocchi, V. D., et al. (2017). Faulty neuronal determination and cell polarization are reverted by modulating HD early phenotypes. PNAS., E762-E771. https://doi.org/ 10.1073/pnas.1715865115.

Publisher's Note Springer Nature remains neutral with regard to jurisdictional claims in published maps and institutional affiliations. 